TRANSACTIONS OF THE

AMERICAN MATHEMATICAL SOCIETY

Volume 349, Number 12, December 1997, Pages 4825-4855

S 0002-9947(97)02040-0

\title{
LEXICOGRAPHIC TAF ALGEBRAS
}

\author{
JUSTIN R. PETERS AND YIU TUNG POON
}

\begin{abstract}
Lexicographic TAF algebras constitute a class of triangular AF algebras which are determined by a countable ordered set $\Omega$, a dimension function, and a third parameter. While some of the important examples of TAF algebras belong to the class, most algebras in this class have not been studied. The semigroupoid of the algebra, the lattice of invariant projections, the Jacobson radical, and for some cases the automorphism group are computed. Necessary and sufficient conditions for analyticity are given. The results often involve the order properties of the set $\Omega$.
\end{abstract}

\section{INTRODUCTION}

This paper treats a class of triangular subalgebras of $\mathrm{AF} C^{*}$-algebras which are called lexicographic TAF algebras and are defined in terms of a countable ordered set, $\Omega$. The familiar examples of the refinement algebra, the standard algebra and the alternation algebra belong to this class; however, most of the TAF algebras in this class have not previously been studied. Although the class is quite broad, the same techniques can be employed on TAF algebras in the class to provide information regarding the semigroupoid of the algebra, the lattice of invariant projections, analyticity, the Jacobson radical, and (in some cases) the automorphism group. The statements of the results often involve the order-properties of the set $\Omega$.

In [Pr 2] and [Pr 3], S. Power studied lexicographic TAF algebras of the form $\mathcal{T}=\underline{\lim }\left(T_{n}, \varphi_{n}\right)$, where $T_{n}$ is the subalgebra of upper triangular $k_{n} \times k_{n}$ complex matrices, and the regular embedding $\varphi_{n}: T_{n} \rightarrow T_{n+1}$ is 'lexicographic'. (See Sec. I.) The class considered here, which generalizes Power's algebras, can be viewed in terms of ordered Bratteli diagrams. Fix a positive integer $L$. The diagram consists of edges connecting the $n^{t h}$ vertex set $V_{n}$ with the $n+1^{s t}, V_{n+1}$. Each $V_{n}$ contains $L$ vertices $v_{0}^{(n)}, \ldots, v_{L-1}^{(n)}$. One can build a diagram as follows: for each $n$ one can choose edges $e$ arbitrarily with source vertex $v_{0}^{(n)}$ and range in $V_{n+1}$. The edges with source $v_{j}^{(n)}$ are then obtained by translation; that is, there is an edge from $v_{j}^{(n)}$ to $v_{k}^{(n+1)}$ if and only if there is an edge from $v_{0}^{(n)}$ to $v_{k-j}^{(n+1)}$ (arithmetic mod $L)$. Multiple edges are allowed; also, the ordering of the edges with source $v_{j}^{(n)}$ is determined by that of the edges with source vertex $v_{0}^{(n)}$. The diagram corresponds to a sequence of finite dimensional triangular algebras $\mathcal{T}_{n}$. The diagonal matrix units in $\mathcal{T}_{n}$ are identified with paths, which are concatenations of edges. These matrix units are ordered in a way determined both by the ordering of the edges which constitute the path, as well as the ordering of the set $\Omega$. (See Sec. II.) The

Received by the editors November 27, 1995.

1991 Mathematics Subject Classification. Primary 46M40, 47D25; Secondary 06F25. 
algebras studied in $[\operatorname{Pr} 2]$ and $[\operatorname{Pr} 3]$ are those for which $L=1$, the single vertex diagrams. Also see [Wa].

Section I introduces the basic notion of a lexicographic inductive system, $(\Omega, \Phi)$. Such systems are characterized in terms of properties of commutative diagrams. In Section II, examples of lexicographic TAF algebras $\mathcal{T}(\Omega, \Phi)$ are presented using ordered Bratteli diagrams. It is shown that there is a general method for constructing lexicographic inductive systems. The semigroupoid $\mathcal{R}(\Omega, \Phi)$, for which an explicit form is given in Section III, may be of interest in its own right. Previously, the only cases in which the semigroupoid has been computed were for certain direct limits of the form $\lim _{\longrightarrow}\left(T_{n}, \varphi_{n}\right)$, where $T_{n}$ is the subalgebra of upper triangular $k_{n} \times k_{n}$ complex matrices. Most of the algebras treated here are more complicated and do not admit such a presentation. Section IV describes the lattice of invariant projections of $\mathcal{T}(\Omega, \Phi)$. Also, the section contains the result that for strongly maximal (not necessarily lexicographic) TAF algebras, if the ambient $\mathrm{AF} C^{*}$-algebra is simple, then $\operatorname{Lat}(\mathcal{T})$ is a nest. Section $V$ addresses the question of which of the algebras $\mathcal{T}(\Omega, \Phi)$ is analytic; in other words, when is there a continuous 1-cocycle on the groupoid $\widetilde{\mathcal{R}}$ of the associated AF $C^{*}$-algebra which is nonnegative exactly on the semigroupoid $\mathcal{R}$ ? The main theorem of this section shows that this is the case if and only if $\Omega$ has one of the order types $\mathbb{Z}^{+}, \mathbb{Z}^{-}$, or $\mathbb{Z}$. Section VI treats the Jacobson radical of $\mathcal{T}(\Omega, \Phi)$. The radical is characterized in terms of a certain conditional expectation (6.6); this generalizes [Pr 2, Theorem 3].

The remaining sections are concerned with isometric automorphisms. In Section VII, the group of outer automorphisms of a TAF algebra $\mathcal{T}$ is identified with Aut $\mathcal{R}$, the automorphism group of the semigroupoid. (A special case of this appears in [Pr 1].) The question of describing Aut $\mathcal{R}(\Omega, \Phi)$ (or Out $\mathcal{T}(\Omega, \Phi)$ ) by explicit parameters seems difficult, if not impossible, for arbitrary inductive systems $(\Omega, \Phi)$. However, for certain $\Omega$, something can be said: for $\Omega=\mathbb{Z}^{+}$or $\mathbb{Z}^{-}$, Aut $\mathcal{R}$ is a finite cyclic group of order $L$ ( $L$ as in the second paragraph). By contrast, we have no results for $\Omega=\mathbb{Z}$ (cf. [Pr 1] for $L=1$ ). Section VIII identifies Aut $\mathcal{R}(\Omega, \Phi)$ with the group of order-preserving, 'dimension-preserving' bijections of $\Omega$ in case $\Omega$ is a dense linear ordering and $L=1$. If $L>1$, the structure of the automorphism group is more complicated. There is always a cyclic automorphism $\tau$ of order $L$. The example in 8.8 shows that the subgroup of Aut $\mathcal{R}$ generated by the order-preserving, dimension-preserving bijections of $\Omega$ and powers of $\tau$ can be proper.

\section{Preliminaries; Notation}

In [Pr 2] and [Pr 3] S. Power studied a class of lexicographic triangular subalgebras of UHF $C^{*}$-algebras and their associated product-type groupoids. We begin by reviewing this class, though our treatment of the material will be different from his.

1.1 Review of Power's lexicographic triangular algebras. Let $\Omega$ be a countable (not finite) linearly ordered set; when convenient, it may be viewed concretely as a subset of $\mathbb{R}$. Let $\eta: \Omega \rightarrow \mathbb{Z}^{+}$be a function with $\eta(\omega) \geq 2, \omega \in \Omega$. We will refer to $\eta$ as a dimension function. If $F \subset \Omega$ is a finite subset, denote $n_{F}=\prod_{\omega \in F} \eta(\omega)$, and $\mathfrak{A}_{F}=M_{n_{F}}$ the $n_{F} \times n_{F}$ complex matrices. If $F=\left\{\omega_{1}, \ldots, \omega_{m}\right\}$, with $\omega_{1}<\omega_{2}<\cdots<\omega_{m}$ ( $\Omega$-ordering), a system of matrix units for $\mathfrak{A}_{F}$ can be given 
as follows: let $[r]=\{0, \ldots, r-1\}$ for any positive integer $r$, and let $I_{F}$ denote the set of all $m$-tuples $\underline{i}=\left(i_{1}, \ldots, i_{m}\right), i_{t} \in\left[\eta\left(\omega_{t}\right)\right], 1 \leq t \leq m$. Let $\left\{e_{\underline{i}, j}^{(F)}, \underline{i}, \underline{j} \in I_{F}\right\}$ be a system of matrix units for $\mathfrak{A}_{F}$. Write $\underline{i} \leq \underline{j}$ if either $\underline{i}=j$ or else for some $t, 1 \leq t \leq m, i_{s}=j_{s}$ for $s<t$ and $i_{t}<j_{t}$. This is the lexicographic order.

Suppose $G$ is a finite subset of $\Omega$ containing $F$, say $G \backslash F=\left\{\omega_{1}^{\prime}, \ldots, \omega_{r}^{\prime}\right\}$. If $\underline{i} \in I_{F}, \underline{i}^{\prime} \in I_{G \backslash F}$ let $\underline{i} \sqcup \underline{i}^{\prime}$ denote the $(m+r)$-tuple whose coordinates belong to either $\underline{i}$ or $\underline{i}^{\prime}$ and are ordered according to the ordering of $\Omega$. In particular, if $r=1$ and $G \backslash F=\left\{\omega^{\prime}\right\}$, then either i) $\omega^{\prime}<\omega_{1}$ or $\quad$ ii) $\omega_{m}<\omega^{\prime}$ or else iii) there is a $t, 1 \leq t<m$, with

$$
\omega_{1}<\cdots<\omega_{t}<\omega^{\prime}<\omega_{t+1}<\cdots<\omega_{m}
$$

Then if $\underline{i}=\left(i_{1}, \ldots, i_{m}\right)$, define

$$
\underline{i} \sqcup s= \begin{cases}\left(s, i_{1}, \ldots, i_{m}\right) & \text { in case i), } \\ \left(i_{1}, \ldots, i_{m}, s\right) & \text { in case ii), } \\ \left(i_{1}, \ldots, i_{t}, s, i_{t+1}, \ldots, i_{m}\right) & \text { in case iii). }\end{cases}
$$

If, as in the paragraph above, $G=F \cup\left\{\omega^{\prime}\right\}$, the embedding $\Phi_{F, G}: \mathfrak{A}_{F} \rightarrow \mathfrak{A}_{G}$ is defined on matrix units by

$$
\Phi_{F, G}\left(e_{\underline{i}, \underline{j}}^{(F)}\right)=\sum_{s \in\left[\eta\left(\omega^{\prime}\right)\right]} e_{\underline{i} \sqcup s, \underline{j} \sqcup s}^{(G)}
$$

for $\underline{i}, \underline{j} \in I_{F}$. Note that $\underline{i} \sqcup s, \underline{j} \sqcup s$ belong to $I_{G}$, so that the sum on the right is indeed a sum of matrix units of $\mathfrak{A}_{G} . \Phi_{F, G}$ is a unital $C^{*}$-embedding. Let $\mathcal{T}_{F}$ (resp., $\mathcal{T}_{G}$ ) denote the upper triangular subalgebra of $\mathfrak{A}_{F}$ (resp., $\mathfrak{A}_{G}$ ); i.e., the span of the matrix units $e_{\underline{i}, \underline{j}}^{(F)}$ (resp., $e_{\underline{i}, \underline{j}}^{(G)}$ ) for which $\underline{i} \leq \underline{j}$. Note that $\Phi_{F, G}$ maps $\mathcal{T}_{F}$ into $\mathcal{T}_{G}$. If $G \backslash F$ is an arbitrary finite set, define $\Phi_{F, G}: \mathfrak{A}_{F} \rightarrow \mathfrak{A}_{G}$ by

$$
\Phi_{F, G}\left(e_{\underline{i}, \underline{j}}^{(F)}\right)=\sum_{\underline{i}^{\prime} \in I_{G \backslash F}} e_{\underline{i} \sqcup \underline{i}^{\prime}, \underline{j} \sqcup \underline{i}^{\prime}}^{(G)} .
$$

It is an easy observation that if $F, G, H$ are finite subsets of $\Omega$ with $F \subset G \subset H$, then $\Phi_{F, H}=\Phi_{G, H} \circ I_{F, G}$.

Observe that if $G \backslash F=\left\{\omega^{\prime}\right\}$ and $\omega^{\prime}<\omega, \omega \in F$, then $\Phi_{F, G}: \mathfrak{A}_{F} \rightarrow \mathfrak{A}_{G}$ is the standard embedding. If $\omega<\omega^{\prime}, \omega \in F$, then $\Phi_{F, G}$ is the refinement embedding. Thus if $\Omega$ is order isomorphic to $\mathbb{Z}^{-}$, then the inductive limit $\mathcal{T}(\Omega, \Phi)=$

$\lim _{\longrightarrow}\left(\mathcal{T}_{F}, \Phi_{F, G}\right)$ is the standard TUHF algebra in the UHF $C^{*}$-algebra of type $F \subset G \subset \Omega$ $\prod \eta(\omega)$. If $\Omega$ is $\mathbb{Z}^{+}, \mathcal{T}(\Omega, \Phi)$ is the refinement TUHF algebra, and if $\Omega$ is $\mathbb{Z}$ it is the alternation algebra [PPW2]. Note, however, that for a general $\Omega$ there is no sequence $\left\{\omega_{n}\right\}_{n=1}^{\infty}$ with $\Omega=\bigcup_{n=1}^{\infty}\left\{\omega_{n}\right\}$ such that the sequential ordering has some prescribed relation to the ordering of $\Omega$.

In summary, given a countable ordered set $\Omega$ and dimension function $\eta$ we have defined a family $\Phi_{F, G}$ of regular $*$-embeddings, a UHF $C^{*}$-algebra $\mathfrak{A}=\mathfrak{A}(\Omega, \Phi)$, and a TUHF algebra $\mathcal{T}=\mathcal{T}(\Omega, \Phi)$. $\mathcal{T}$ is a direct limit of triangular subalgebras of the form $\mathcal{T}_{F}$ which is maximal triangular in the factor $\mathfrak{A}_{F}$. Let $\mathcal{D}=\mathcal{D}(\Omega, \Phi)=\mathcal{T} \cap \mathcal{T}^{*}$ be the canonical masa of $\mathcal{T}$. The maximal ideal space of $\mathcal{D}$ is homeomorphic with 
the product space

$$
X=\prod_{\omega \in \Omega}[\eta(\omega)] .
$$

Associated with the pair $(\mathfrak{A}, \mathcal{D})$ is the groupoid $\widetilde{\mathcal{R}}$, which is a subset of $X \times X$ consisting of all pairs $(x, y)$ with $x_{\omega}=y_{\omega}$ for all but finitely many $\omega \in \Omega$. The topology on $\widetilde{\mathcal{R}}$ (which is not the product topology $X \times X$ restricted to $\mathcal{R}$ ) is obtained by declaring an open basis as follows: let $F \subset \Omega$ be a finite set, and let $\underline{i}, \underline{j} \in I_{F}$. Let

$$
\widehat{e}_{\underline{i}, \underline{j}}^{(F)}=\left\{(x, y) \in \widetilde{\mathcal{R}}: x_{\omega}=i_{\omega}, y_{\omega}=j_{\omega}, \omega \in F\right\} .
$$

Then $\left\{\widehat{e}_{\underline{i}, \underline{j}}^{(F)}: F\right.$ finite, $\left.\underline{i}, \underline{j} \in I_{F}\right\}$ is a basis for $\widetilde{\mathcal{R}}$. One can show that each $\widehat{e}_{\underline{i}, \underline{j}}^{(F)}$ is also closed and compact.

The semigroupoid $\mathcal{R}$ associated with the triangular UHF algebra $\mathcal{T}$ is given by $\mathcal{R}=\left\{(x, y) \in \widetilde{\mathcal{R}}:(x, y) \in \widehat{e}_{\underline{i}, \underline{j}}^{(F)}\right.$ for some finite $F \subset \Omega, \underline{i}, \underline{j} \in I_{F}$ with $\left.\underline{i} \leq \underline{j}\right\}$

Note that if $(x, y) \in \widehat{e}_{\underline{i}, \underline{j}}^{(F)}$ for some $F, \underline{i}, \underline{j}$, then for every $G \supset F$, there exists $\underline{i}^{\prime} \in I_{G \backslash F}$ such that $(x, y) \in \widehat{e}_{\underline{i} \sqcup \underline{i}^{\prime}, \underline{j} \sqcup \underline{i}^{\prime}}^{(G)}$ and we have $\underline{i} \leq \underline{j}$ iff $\underline{i} \sqcup \underline{i}^{\prime} \leq \underline{j} \sqcup \underline{i}^{\prime}$ Our goal is to generalize Power's construction to the case of $\mathrm{AF} C^{*}$-algebras which are direct limits of subalgebras $\mathfrak{A}_{F}$, where the $\mathfrak{A}_{F}$ are direct sums of factors.

1.2 Definition of lexicographic TAF algebras. Let $\Omega$ be a countable ordered set, $\eta: \Omega \underset{L-1}{\rightarrow} \mathbb{Z}(\eta(\omega) \geq 2, \omega \in \Omega)$ a dimension function, and $L \geq 1$ an integer. Set $\mathfrak{A}_{F}=\bigoplus_{l=0}^{L-1} \mathfrak{A}_{F, l}$, where $\mathfrak{A}_{F, l} \cong \mathfrak{A}_{F, k}(k, l \in[L])$ is a finite dimensional factor isomorphic to $M_{n_{F}}, n_{F}=\prod_{\omega \in F} \eta(\omega)$, for $F$ a finite subset of $\Omega$.

Suppose for each finite subset $F \subset \Omega$ we have fixed an isomorphism of $\mathfrak{A}_{F}$ with $M_{n_{F}} \oplus \cdots \oplus M_{n_{F}}$ ( $L$ summands). Then we define $\tau_{F}$ to be the automorphism of $\mathfrak{A}_{F}$ which is conjugate to the cyclic permutation $\left(a_{0}, \ldots a_{L-1}\right) \rightarrow\left(a_{L-1}, a_{0}, \ldots, a_{L-2}\right)$ of $M_{n_{F}} \oplus \cdots \oplus M_{n_{F}}$.

Assume that for each pair of finite subsets $F \subset G$ of $\Omega$ there is a $C^{*}$-embedding $\Phi_{F, G}: \mathfrak{A}_{F} \rightarrow \mathfrak{A}_{G}$ satisfying:

$$
\begin{aligned}
& \text { for any } F \subset G \subset H \subset \Omega \text {, the diagram } \\
& \begin{array}{rlrl}
\mathfrak{A}_{F} & \stackrel{\mathrm{id}}{\longrightarrow} & \mathfrak{A}_{F} & \\
\Phi_{F, G} \downarrow & & & \\
\mathfrak{A}_{G} & \underset{\Phi_{F, H}}{ } & & \text { commutes; } \\
& \mathfrak{A}_{H} &
\end{array}
\end{aligned}
$$

and

for any $F \subset G \subset \Omega$, the diagram

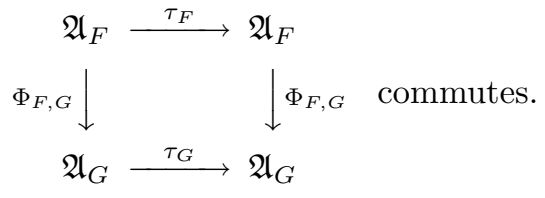


(I.1) is just the condition that $\left\{\left(\mathfrak{A}_{F}, \Phi_{F, G}\right): F \subset G \subset \Omega\right\}$ forms an inductive system, and so the inductive limit algebra $\mathfrak{A}=\mathfrak{A}(\Omega, \Phi)=\underset{F \subset G}{\underset{\lim }{\longrightarrow}}\left(\mathfrak{A}_{F}, \Phi_{F, G}\right)$, which is an $\mathrm{AF} C^{*}$-algebra, can be formed. (I.2) implies there is an automorphism, which we will denote by $\tau$, of $\mathfrak{A}$, such that $\tau$ restricted to $\mathfrak{A}_{F}$ is precisely $\tau_{F}$. Let $\mathfrak{A}_{F}^{\tau}$ denote the $\tau$-fixed subalgebra of $\mathfrak{A}_{F}$, i.e., $\mathfrak{A}_{F}^{\tau}=\{a \in \mathfrak{A}: \tau(a)=a\}$. (I.2) implies that $\Phi_{F, G}$ maps $\mathfrak{A}_{F}^{\tau}$ into $\mathfrak{A}_{G}^{\tau}$. (For convenience, we let $\Phi_{F, G}$ also denote the restriction of $\Phi_{F, G}$ to $\mathfrak{A}_{F}^{\tau}$.) It is evident that $\mathfrak{A}_{F}^{\tau} \cong M_{n_{F}}$. We now further assume that

$$
\Phi_{F, G}: \mathfrak{A}_{F}^{\tau} \rightarrow \mathfrak{A}_{G}^{\tau}
$$

is a lexicographic embedding of factors of the type studied by Power, as described earlier.

Let $\left\{e_{\underline{i}, j}^{(F)}: \underline{i}, \underline{j} \in I_{F}\right\}$ be a system of matrix units for $\mathfrak{A}_{F}^{\tau}$, and let $e^{(F, k)}$ denote the central projection in $\mathfrak{A}_{F}$ onto the factor $\mathfrak{A}_{F, k}, k \in[L]$. Then $e_{\underline{i}, \underline{j}}^{(F, k)}:=e^{(F, k)} e_{\underline{\underline{i}}, \underline{\underline{j}}}^{(F)}$ $\left(\underline{i}, \underline{j} \in I_{F}, k \in[L]\right)$ is a system of matrix units for $\mathfrak{A}_{F}$. Let $\mathcal{T}_{F}^{\tau}$ (resp., $\mathcal{T}_{F, k}$ ) denote the span of the upper triangular matrix units $e_{\underline{i}, \underline{j}}^{(F)}$ with $\underline{i} \leq \underline{j}$ of $\mathfrak{A}_{F}^{\tau}$ (resp., $e_{\underline{i}, \underline{j}}^{(F, k)}$ with $\underline{i} \leq \underline{j}, k \in[L]$ of $\mathfrak{A}_{F, k}$ ), and $\mathcal{T}_{F}:=\bigoplus_{k \in[L]} \mathcal{T}_{F, k}$. If $F \subset G \subset \Omega$, the embedding $\Phi_{F, G}$ maps $\mathcal{T}_{F}^{\tau}$ into $\mathcal{T}_{G}^{\tau}$ by (1.2). Hence $\Phi_{F, G}$ maps a matrix unit $e_{\underline{i}, \underline{j}}^{(F, k)}$ in $\mathfrak{A}_{F}$ with $\underline{i} \leq \underline{j}$ into sums of upper triangular matrix units $e_{{\underline{i^{\prime}}}^{\prime}, \underline{j}^{\prime}}^{(G, \ell)}$ in $\mathfrak{A}_{G}$ with $\underline{i}^{\prime} \leq \underline{j^{\prime}}$. Thus $\Phi_{F, G}\left(\mathcal{T}_{F}\right) \subset \mathcal{T}_{G}$. We can now define $\mathcal{T}(\Omega, \Phi)=\underset{F \subset G \subset \Omega}{\lim _{\longrightarrow}}\left(\mathcal{T}_{F}, \Phi_{F, G}\right) . \mathcal{T}(\Omega, \Phi)$ is a strongly maximal, lexicographic $T A F$ algebra.

1.2.1 Definition. Let $\Omega$ be a countable ordered set. and $\Phi=\left\{\Phi_{F, G}: F \subset G \subset \Omega\right\}$ a collection of $C^{*}$-embeddings satisfying (I.1), (I.2) and (I.3). The pair $(\Omega, \Phi)$ will subsequently be referred to as a lexicographic inductive system, or simply as an inductive system. Implicit in $\Phi$ are a positive integer $L=L(\Phi)$ and a dimension function $\eta=\eta(\Phi)$, such that for each finite subset $F$ of $\Omega$ we have $\mathfrak{A}_{F}, \mathfrak{A}_{F}^{\tau}$ and $\tau_{F}$ as above.

If $(\Omega, \Phi)$ is an inductive system with $L=1$, then the automorphism $\tau$ is the identity, and $\mathcal{T}^{\tau}(\Omega, \Phi)=\mathcal{T}(\Omega, \Phi)$ is a lexicographic TUHF algebra of the type studied by Power. Of course, one can obtain inductive systems with $L>1$ by taking direct sums of systems with $L=1$. In the next section we will see how to construct 'nontrivial' inductive systems with $L>1$.

\section{A General Construction}

Suppose we are given a countable ordered set $\Omega$, an integer $L \geq 1$, and a dimension function $\eta$. We will exhibit a general method for constructing a system of embeddings $\Phi=\left\{\Phi_{F, G}: F \subset G \subset \Omega\right\}$ such that $(\Omega, \Phi)$ is a lexicographic inductive system with $L=L(\Phi)$ and $\eta=\eta(\Phi)$.

Let $\nu: \Omega(\eta)=\{(\omega, s): \omega \in \Omega, \quad s \in[\eta(\omega)]\} \rightarrow[L]$ be an arbitrary function. We will define a system of embeddings $\Phi^{\nu}$ as follows. Let $F$ be any finite subset of $\Omega$, and $\omega \in \Omega \backslash F, G=F \cup\{\omega\}$. If $e_{\underline{i}, \underline{j}}^{(F, k)}\left(k \in[L], \underline{i}, \underline{j} \in I_{F}\right)$ is a matrix unit of $\mathfrak{A}_{F}$, set

$$
\Phi_{F, G}^{\nu}\left(e_{\underline{i}, \underline{j}}^{(F, k)}\right)=\sum_{s \in[\eta(\omega)]} e_{\underline{i} \sqcup s, \underline{j} \sqcup s s}^{(G, k+\nu(\omega, s))} .
$$


If $G$ is any finite subset of $\Omega$ containing $F$, set $\Phi_{F, G}^{\nu}$ to be a composition of embeddings $\Phi_{F^{\prime}, G^{\prime}}^{\nu}$ where card $\left(G^{\prime} \backslash F^{\prime}\right)=1$. While there are various ways this can be done, one nevertheless checks that the resulting $\Phi_{F, G}^{\nu}$ is uniquely defined. Thus the system $\Phi^{\nu}$ satisfies (I.1). It is straightforward to check that $\Phi^{\nu}$ satisfies (I.2) and (I.3) as well, so that $\left(\Omega, \Phi^{\nu}\right)$ is a lexicographic inductive system.

Let $\left\{\omega_{n}\right\}_{n=1}^{\infty}$ be a sequence with $\Omega=\bigcup_{n=1}^{\infty}\left\{\omega_{n}\right\}$. Set $F_{n}=\left\{\omega_{1}, \ldots, \omega_{n}\right\}, \mathfrak{A}_{n}=\mathfrak{A}_{F_{n}}$ and $\Phi_{n}=\Phi_{F_{n}, F_{n+1}}$. The embedding $\Phi_{n}: \mathfrak{A}_{n} \rightarrow \mathfrak{A}_{n+1}$ can be described by an ordered Bratteli diagram (cf. [HPS], [Po Wa]). Furthermore, it is evident from the formula (II.1) that $\Phi_{n}^{\nu}(e)$ for any matrix unit $e \in \mathfrak{A}_{n}$ is determined by knowing how diagonal matrix units embed. A diagram is said to be stationary if the number and ordering of the edges from the $n^{t h}$ vertex set to the $(n+1)^{s t}$ vertex set is independent of $n$.

\subsection{Examples.}

2.1.1 Let $\Omega$ be any countable ordered set, $L=2, \eta(\omega)=2, \omega \in \Omega$, and set $\nu(\omega, s)=$ 0 for all $\omega \in \Omega, s \in[2]$. Given any sequence $\left\{\omega_{n}\right\}_{n=1}^{\infty}$ with $\Omega=\bigcup_{n=1}^{\infty}\left\{\omega_{n}\right\}$, the embeddings $\Phi_{n}$ can be described by the stationary ordered Bratteli diagram

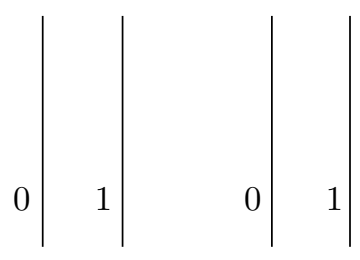

The AF $C^{*}$-algebra $\mathfrak{A}\left(\Omega, \Phi^{\nu}\right)$ is the direct sum of two copies of $\operatorname{UHF}\left(2^{\infty}\right)$, and the TAF algebra $\mathcal{T}\left(\Omega, \Phi^{\nu}\right)$ is the direct sum of two copies of Power's lexicographic algebras.

2.1.2. $\Omega, L, \eta,\left\{\omega_{n}\right\}_{n=1}^{\infty}$ as above, but now take $\nu(\omega, s)=s, \omega \in \Omega, s \in[2]$. This yields the stationary diagram

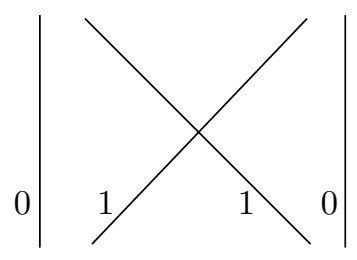

The role of the sequence $\left\{\omega_{n}\right\}_{n=1}^{\infty}$ is illustrated in Example 2.1.5. It should 
be emphasized, however, that neither $\underset{\lim }{\longrightarrow}\left(\mathfrak{A}_{n}, \Phi_{n}\right)$ nor $\underline{\lim }\left(\mathcal{T}_{n}, \Phi_{n}\right)$ depends on the particular sequence.

2.1.3. With $\Omega, L, \eta$ and $\left\{\omega_{n}\right\}_{n=1}^{\infty}$ as above, set $\nu(\omega, s)=1-s(\bmod 2), \omega \in \Omega, s \in[2]$. The embeddings $\Phi_{n}$ yield the stationary diagram

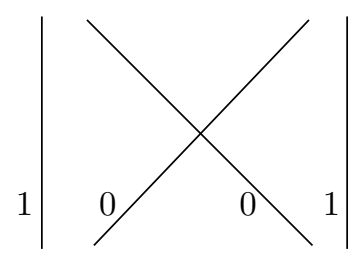

2.1.4. With $\Omega, L, \eta,\left\{\omega_{n}\right\}_{n=1}^{\infty}$ as before, the only remaining stationary choice of $\nu$ is $\nu(\omega, s)=1, \omega \in \Omega, s \in[2]$. This gives the diagram

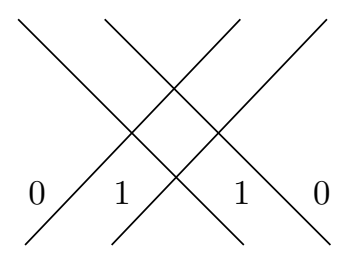

The inductive limit $\mathcal{T}\left(\Omega, \Phi^{\nu}\right)$ is isomorphic to that of Example 1. (See Proposition 2.4.)

Of course, non-stationary diagrams can be obtained by allowing $\nu$ to vary with $\omega \in \Omega$.

2.1.5. With $\Omega, L,\left\{\omega_{n}\right\}_{n=1}^{\infty}$ as before, let the dimension function $\eta$ be $\eta(\omega)=3, \omega \in$ $\Omega$, and define $\nu(\omega, s)=s(\bmod 2), \omega \in \Omega, s \in[3]$. We obtain the stationary diagram

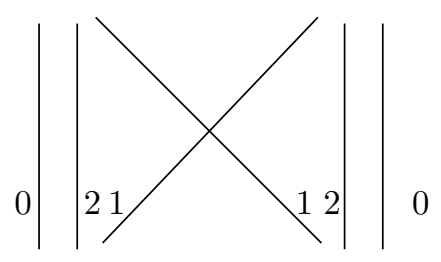

In this case, the factors in $\mathfrak{A}_{n}$ partially embed into the factors of $\mathfrak{A}_{n+1}$ with different multiplicities. Let $p_{j}^{(i)}$ denote the edge having as range the vertex on the left if $i=0$, and on the right if $i=1$, with label $j$. Diagonal matrix units in $\mathfrak{A}_{n}$ can be realized as concatenations of edges. Consider $n=3$ : 


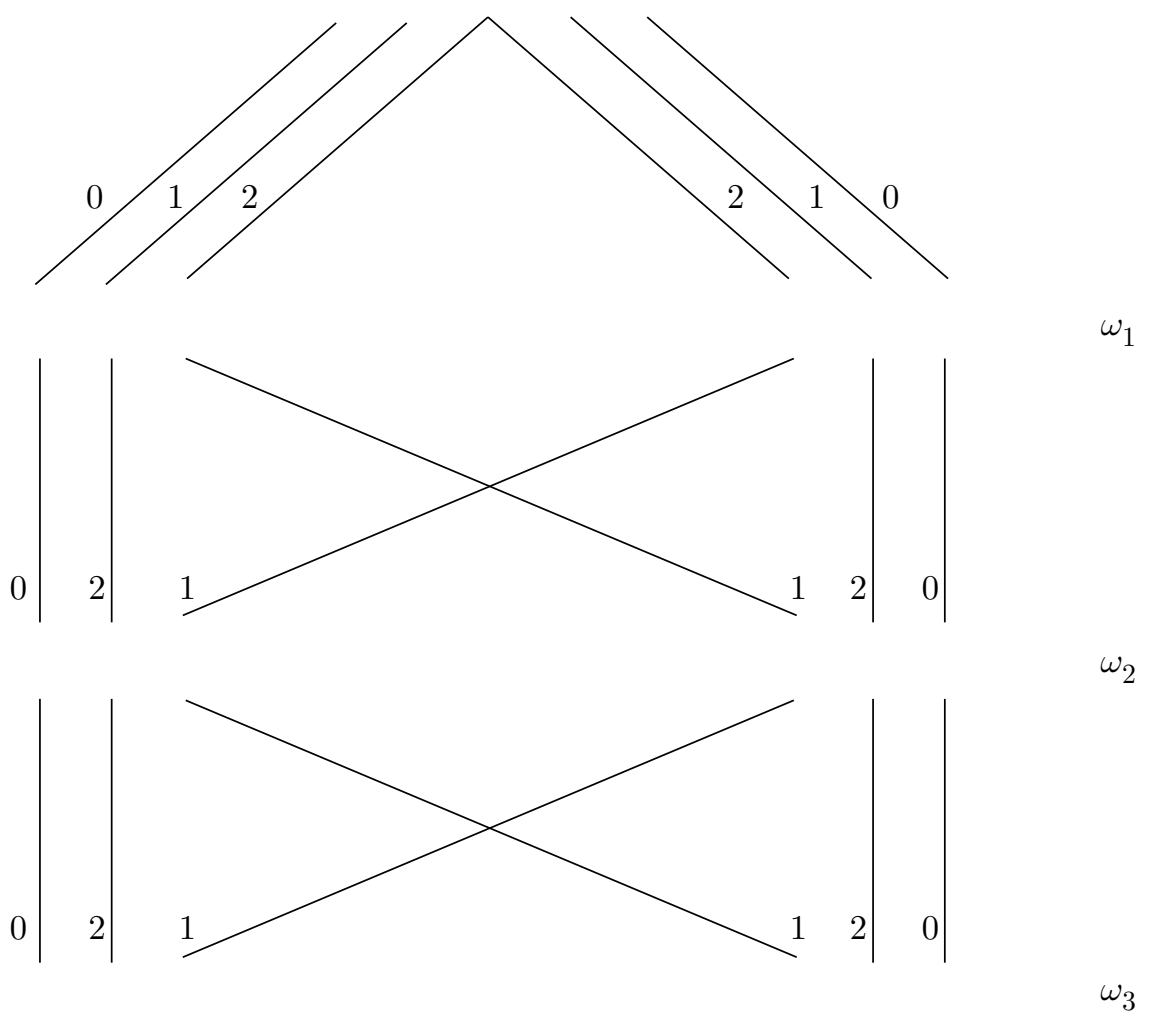

Notice there are 27 distinct paths from the topmost vertex to the bottom left vertex, and another 27 paths from the top vertex to the bottom right vertex. These correspond to diagonal matrix units in the factors $\mathfrak{A}_{3,0}, \mathfrak{A}_{3,1}$ respectively. Similarly, paths from the top vertex to the $\omega_{2}$-level represent diagonal matrix units in $\mathfrak{A}_{2}$. Suppose, in the $\Omega$-ordering, $\omega_{2}<\omega_{1}<\omega_{3}$. The diagonal matrix unit $e_{(0,1)}^{(2,0)}$ in $\mathfrak{A}_{2,0}$ is identified with the path $p_{1}^{(0)} p_{0}^{(0)}$. As $\omega_{2}<\omega_{1}$, the subscript $(0,1)$ of the matrix units indicates a ' 0 ' in the $\omega_{2}$-position and ' 1 ' in the $\omega_{1}$-position. Applying $\Phi_{2}$, obtain $\Phi_{2}\left(e_{(0,1)}^{(2,0)}\right)=$ $e_{(0,1,0)}^{(3,0)}+e_{(0,1,1)}^{(3,1)}+e_{(0,1,2)}^{(3,0)}$. These three matrix units are identified with the paths

$$
p_{1}^{(0)} p_{0}^{(0)} p_{0}^{(0)}, \quad p_{1}^{(0)} p_{0}^{(0)} p_{1}^{(1)}, \quad p_{1}^{(0)} p_{0}^{(0)} p_{2}^{(0)}
$$

respectively. Note that as $\omega_{3}$ follows both $\omega_{1}$ and $\omega_{2},(0,1) \sqcup s=(0,1, s)$.

Suppose, however that $\omega_{2}<\omega_{3}<\omega_{1}$. In this case, $\Phi_{2}$ embeds as

$$
\Phi_{2}\left(e_{(0,1)}^{(2,0)}\right)=e_{(0,0,1)}^{(3,0)}+e_{(0,1,1)}^{(3,1)}+e_{(0,2,1)}^{(3,0)} .
$$

Here, $(0,1) \sqcup s=(0, s, 1)$. The matrix unit summands are identified with the paths

$$
p_{1}^{(0)} p_{0}^{(0)} p_{0}^{(0)}, p_{1}^{(0)} \quad p_{0}^{(0)} p_{1}^{(1)}, \quad p_{1}^{(0)} p_{0}^{(0)} p_{2}^{(0)}
$$

respectively. This illustrates the role of the sequence $\left\{\omega_{n}\right\}_{n=1}^{\infty}$ in the embeddings. 
2.1.6. With $\Omega,\left\{\omega_{n}\right\}_{n=1}^{\infty}$ as before, let $L=3, \eta(\omega)=3, \omega \in \Omega$ and set $\nu(\omega, s)=$ $s, \omega \in \Omega, s \in[3]$. This yields the stationary diagram

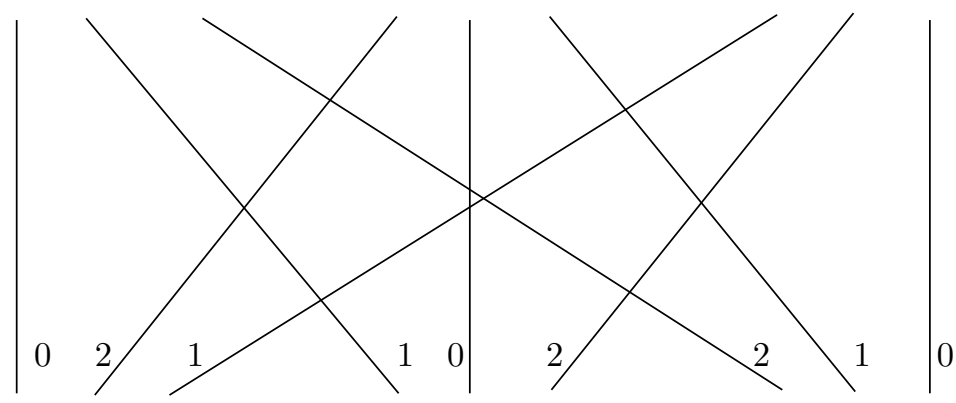

2.2 Theorem. Every lexicographic inductive system $(\Omega, \Phi)$ is equivalent to one of the form $\left(\Omega, \Phi^{\nu}\right)$. In other words, given $(\Omega, \Phi)$ and $L=L(\Phi), \eta=\eta(\Phi)$, there is a function $\nu: \Omega(\eta) \rightarrow[L]$, with $\Omega(\eta)$ and $\Phi^{\nu}$ as defined at the beginning of this section, such that

$$
\mathfrak{A}(\Omega, \Phi) \cong \mathfrak{A}\left(\Omega, \Phi^{\nu}\right) \text { and } \mathcal{T}(\Omega, \Phi) \cong \mathcal{T}\left(\Omega, \Phi^{\nu}\right) .
$$

Proof. We observed earlier that for any function $\nu$ the embeddings $\Phi^{\nu}$ given by (II.1) form an inductive system. Suppose now an inductive system $(\Omega, \Phi)$ is given. Let $\left\{\omega_{n}\right\}_{n=1}^{\infty}$ be any sequence in $\Omega$ with $\Omega=\bigcup_{n=1}^{\infty}\left\{\omega_{n}\right\}$, and set $F_{n}=\left\{\omega_{1}, \ldots, \omega_{n}\right\}$, $\mathfrak{A}_{n}=\mathfrak{A}_{F_{n}}, \Phi_{n}=\Phi_{F_{n}, F_{n+1}}$ and $I_{n}=I_{F_{n}}$ (index set). As in Section I, we obtain a system of matrix units $\left\{e_{\underline{i}, \underline{j}}^{(n, l)}: l \in[L], \underline{i}, \underline{j} \in I_{n}\right\}$ for $\mathfrak{A}_{n}$. There are integers $l_{s} \in[L]$ for $s \in\left[\eta\left(\omega_{n+1}\right)\right]$ such that

$$
\Phi_{n}\left(e_{\underline{i}, \underline{j}}^{(n, 0)}\right)=\sum_{s \in\left[\eta\left(\omega_{n+1}\right)\right]} e_{\underline{i} \sqcup s, \underline{j} \sqcup s}^{\left(n+1, l_{s}\right)} .
$$

Because $\Phi_{n}$ is multiplicative, the integers $l_{s}$ do not depend on the indices $\underline{i}, \underline{j} \in I_{n}$.

Define $\nu\left(\omega_{n+1}, s\right)=l_{s}, s \in\left[\eta\left(\omega_{n+1}\right)\right]$. The condition (I.2) implies that $\Phi_{n}$ is determined by how the factor $\mathfrak{A}_{n, 0}$ partially embeds in $\mathfrak{A}_{n+1}$. Thus

$$
\begin{aligned}
\Phi_{n}\left(e_{\underline{i}, \underline{j}}^{(n, k)}\right) & =\tau^{k} \circ \Phi_{n} \circ \tau^{-k}\left(e_{\underline{i}, \underline{j}}^{(n, k)}\right) \\
& =\tau^{k}\left(\Phi_{n}\left(e_{\underline{i}, \underline{j}}^{(n, 0)}\right)\right) \\
& =\tau^{k}\left(\sum_{s \in\left[\eta\left(\omega_{n+1}\right)\right]} e_{\left.\underline{i} \sqcup s, \underline{j} \sqcup s^{\left(n+1, \nu\left(\omega_{n+1}, s\right)\right)}\right)}\right) \\
& =\sum_{s \in\left[\eta\left(\omega_{n+1}\right)\right]} e_{\underline{i} \sqcup s, \underline{j} \sqcup s}^{\left(n+1, k+\nu\left(\omega_{n+1}, s\right)\right)} \\
& =\Phi_{n}^{\nu}\left(e_{\underline{i}, \underline{j}}^{(n, k)}\right) .
\end{aligned}
$$

We need only to define $\nu\left(\omega_{1}, s\right)$. If $F$ is any finite subset of $\Omega$ not containing $\omega_{1}, G=F \cup\left\{\omega_{1}\right\}$, then the values $\nu\left(\omega_{1}, s\right)$ are obtained from $\Phi_{F, G}\left(e_{\underline{i}, \underline{j}}^{(F, 0)}\right)$ as 
above. We now have two systems $\left\{\left(\mathfrak{A}, \Phi_{F, G}\right)\right\}$ and $\left\{\left(\mathfrak{A}, \Phi_{F, G}^{\nu}\right)\right\}$ which satisfy (I.1), the condition for consistency.

As they agree on an increasing sequence $F=F_{n}, G=F_{n+1}$, it follows that

$$
\underset{F \subset G \subset \Omega}{\lim _{F}}\left\{\left(\mathfrak{A}_{F}, \Phi_{F, G}\right)\right\}=\underset{F \subset G \subset \Omega}{\lim _{\vec{C}}}\left\{\left(\mathfrak{A}_{F}, \Phi_{F, G}^{\nu}\right)\right\} .
$$

A similar statement holds for $\mathcal{T}_{F}$ in place of $\mathfrak{A}_{F}$.

2.3 Remarks. 1) While we have constructed $\nu$ so that $\Phi_{F, G}^{\nu}=\Phi_{F, G}$ for $F=$ $F_{n}, G=F_{n+1}$, we have not proved equality for arbitrary $F \subset G \subset \Omega$. Nevertheless, we know that the inductive limits with respect to $\Phi, \Phi^{\nu}$ both exist, so that the limit does not depend on the choice of the sequence $F_{n}$. That is sufficient for the conclusion. It may in fact be true that $\Phi_{F, G}=\Phi_{F, G}^{\nu}$ for all $F \subset G \subset \Omega$, though it has not been verified.

2) The proof suggests how to construct a lexicographic TAF algebra $\mathcal{T}(\Omega, \Phi)$ by building a Bratteli diagram. Choose a sequence $\left\{\omega_{n}\right\}_{n=1}^{\infty}$ which fills out $\Omega$, and choose $L \geq 1$. Let $\mathcal{V}=\bigcup_{n=0}^{\infty} V_{n}$ be the set of vertices, with $V_{0}=\left\{v_{0}^{0}\right\}, V_{n}=$ $\left\{v_{n}^{0}, \ldots, v_{n}^{L-1}\right\}, n \geq 1$. There must be the same number of edges from $v_{0}^{0}$ to each $v_{1}^{i}, i \in[L]$, and that number determines $\eta\left(\omega_{1}\right)$. There is, clearly, only one way of range-ordering the edges (up to order isomorphism). In building the diagram from $V_{1}$ to $V_{2}$, one has freedom to choose any number of edges with range $v_{2}^{0}$, and order them arbitrarily. However, the number and ordering of edges with range $v_{2}^{i}$ is determined by 'shifting' $(\bmod L)$ the edges into $v_{2}^{0}$. This determines $\eta\left(\omega_{2}\right)$ as well as $\nu\left(\omega_{2}, s\right), s \in\left[\eta\left(\omega_{2}\right)\right]$. One continues in this way to construct the diagram. Of course, if the diagram is stationary, there are no more choices to be made.

2.4 Proposition. Let $\Omega$ be a countable ordered set, $\eta: \Omega \rightarrow \mathbb{Z}^{+}$a dimension function, and $L$ a positive integer. Suppose $\nu, \mu$ are two functions from $\Omega(\eta)$ to $[L]$ such that the difference $\nu(\omega, s)-\mu(\omega, s)$ depends only on $\omega$. Then

$$
\mathcal{T}\left(\Omega, \Phi^{\nu}\right) \cong \mathcal{T}\left(\Omega, \Phi^{\mu}\right) .
$$

Proof. Let $\left\{\omega_{n}\right\}_{n=1}^{\infty}$ be a sequence in $\Omega$ with $\Omega=\bigcup_{n=1}^{\infty}\left\{\omega_{n}\right\}$. Set $F_{n}=\left\{\omega_{1}, \ldots, \omega_{n}\right\}$, $\mathfrak{A}_{n}=\mathfrak{A}_{F_{n}}$, and $\Phi_{n}^{\alpha}=\Phi_{F_{n}, F_{n+1}}^{\alpha}$ for $\alpha=\mu, \nu$. Define sequences $\left\{k_{n}\right\}_{n=1}^{\infty},\left\{l_{n}\right\}_{n=1}^{\infty}$ by $k_{n}=\mu\left(\omega_{n}, 0\right)-\nu\left(\omega_{n}, 0\right)$ and $l_{n}=k_{1}+\cdots+k_{n}, n=1,2, \ldots$ With $\mathfrak{A}_{n}=\bigoplus_{k=0}^{L-1} \mathfrak{A}_{n, k}$, define $\mathcal{B}_{n, k}=\mathfrak{A}_{n, k+l_{n}}$, and $\mathcal{B}_{n}=\bigoplus_{k=0}^{L-1} \mathcal{B}_{n, k}$. Define $\varphi_{n}: \mathfrak{A}_{n} \rightarrow \mathcal{B}_{n}$ by $\varphi_{n}\left(e_{\underline{i}, \underline{j}}^{(n, k)}\right)=$ $e_{\underline{i}, \underline{j}}^{\left(n, k+l_{n}\right)}$. One checks that the diagram

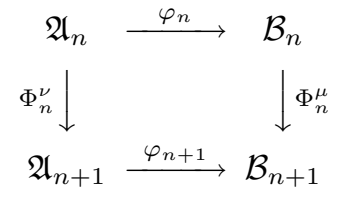

commutes; also if $\varphi_{n}, \varphi_{n+1}$ are replaced by their inverses, the diagram commutes. Finally, replacing $\mathfrak{A}_{n}, \mathcal{B}_{n}$ by the upper triangular subalgebras, the diagram still commutes, and yields the desired conclusion. 
2.5 Remark. As a result of Proposition 2.4, we may assume that the function $\nu$ satisfies $\nu(\omega, 0)=0, \omega \in \Omega$. In this case we will say that the diagram is (or, the embeddings are) in standard form.

\section{The Semigroupoid $\mathcal{R}(\Omega, \Phi)$}

Let $(\Omega, \Phi)$ be a lexicographic inductive system. Let

$$
\mathcal{D}=\mathcal{D}(\Omega, \Phi)=\mathcal{T}(\Omega, \Phi) \cap \mathcal{T}(\Omega, \Phi)^{*}
$$

be the canonical masa of the TAF algebra $\mathcal{T}(\Omega, \Phi)$, and let $X=\widehat{\mathcal{D}}$ be its maximal ideal space.

Let $\widetilde{\mathcal{R}}$ be the $r$-discrete principal groupoid associated with the AF $C^{*}$-algebra $\mathfrak{A}$ and canonical masa $\mathcal{D}$, and $\mathcal{R}$ the semigroupoid associated with $\mathcal{T}$. Since $\mathcal{T}$ is strongly maximal triangular in $\mathfrak{A}, \mathcal{R} \cap \mathcal{R}^{-1}=\Delta(X)=\{(x, x): x \in X\}$, and $\mathcal{R} \cup \mathcal{R}^{-1}=\widetilde{\mathcal{R}}$

If $L>1$, let $\mathcal{T}_{F}^{\tau}$ be the $\tau$-fixed subalgebra of $\mathcal{T}_{F} \subset \mathfrak{A}_{F}$ as described in 1.2 and $\mathcal{T}^{\tau}=\mathcal{T}^{\tau}(\Omega, \Phi)=\varliminf_{\mathfrak{l}}\left\{\left(\mathcal{T}_{F}^{\tau}, \Phi_{F, G}\right): F \subset G \subset \Omega\right\}$ (where $\Phi_{F, G}$ is used to denote the embedding $\mathfrak{A}_{F} \hookrightarrow \mathfrak{A}_{G}$ as well as any of the restrictions $\mathcal{T}_{F} \hookrightarrow \mathcal{T}_{G}, \mathcal{D}_{F} \hookrightarrow \mathcal{D}_{G}, \mathcal{T}_{F}^{\tau} \hookrightarrow$ $\mathcal{T}_{G}^{\tau}$, or $\mathcal{D}_{F}^{\tau} \hookrightarrow D_{G}^{\tau}$. It should be clear from context which is meant.) Then the maximal ideal space $X^{\tau}$ of $\mathcal{D}^{\tau}$ is identified with $\prod_{\omega \in \Omega}[\eta(\omega)]$, and the groupoid $\widetilde{\mathcal{R}}^{\tau}$ (resp., semigroupoid $\mathcal{R}^{\tau}$ ) associated with $\mathfrak{A}^{\tau}$ (resp., $\mathcal{T}^{\tau}$ ) is as described in 1.1. We are going to describe the groupoid $\widetilde{\mathcal{R}}$ and show how it is related to $\widetilde{\mathcal{R}}^{\tau}$.

3.1 Explicit description of the semigroupoid. Given a finite subset $F$ of $\Omega$, $\underline{i} \in I_{F}$ and $k \in[L]$, let $\left\{e_{\underline{i}}^{(F, k)}, \underline{i}, \underline{j} \in I_{F}, k \in[L]\right\}$ be a system of matrix units as described in Section 1.2. Then every $e_{\underline{i} j}^{(F, k)}$ corresponds to a subset $\left\{\widehat{e}_{\underline{i}}^{(F)}\right\}$ of $\mathcal{R}^{\tau}$. For simplicity of notation, we will write $e_{\underline{i}}^{(F, k)}$ and $\widehat{e}_{\underline{i}}^{(F, k)}$ for $e_{\underline{i}}^{(F, k)}$ and $\widehat{e}_{\underline{i}}^{(F, k)}$ respectively.

Suppose $F \subset G$ are finite subsets of $\Omega$. For every $\underline{i} \in I_{F}, \underline{j} \in I_{G \backslash F}$, we have that $\Phi_{F, G}\left(e_{\underline{i}}^{(F, k)}\right)$ dominates $e_{\underline{i} \cup \underline{j}}^{\left(G, k^{\prime}\right)}$, where $k^{\prime}=k+\sum_{\omega \in G \backslash F} \nu\left(\omega, i_{\omega}\right)$, and the sum is taken $\bmod L$.

Let $\left(F_{n}\right)_{n=1}^{\infty}$ be an increasing sequence of finite subsets of $\Omega, \bigcup_{n=1}^{\infty} F_{n}=\Omega$. Any point $x \in X$ can be expressed as $\{x\}=\bigcap_{n=1}^{\infty} \widehat{e}_{\underline{i}_{n}}^{\left(F_{n}, k_{n}\right)}$, for some suitable $\underline{i}_{n} \in I_{F_{n}}$ and $k_{n} \in[L]$ satisfying $k_{n+1}=k_{n}+\sum_{\omega \in F_{n+1} \backslash F_{n}} \nu\left(\omega, i_{\omega}\right)$. Therefore, $k=k_{n}-\sum_{\omega \in F_{n}} \nu\left(\omega, i_{\omega}\right)$ is independent of $n$. Thus, we can write $X=\bigcup_{k \in[L]} X_{k}$, where

$$
X_{k}=\left\{(k, \underline{i}): \underline{i}=\left(i_{\omega}\right)_{\omega \in \Omega} \in \prod_{\omega \in \Omega}[\eta(\omega)]\right\} .
$$

Let $x=(k, \underline{i}) \in X$. We will write $k(x)$ for $k$ and $x_{\omega}$ for $i_{\omega}$. For every finite subset $F$ of $\Omega$, we have $x \in \widehat{e}_{\underline{i}^{\prime}}^{\left(F, k^{\prime}\right)}$, where $k^{\prime}=k(x)+\sum_{\omega \in F} \nu\left(\omega, x_{\omega}\right)$. It follows that $X$ is homeomorphic to $[L] \times X^{\tau}$. 
3.1.1 Proposition. Suppose $F \subset \Omega$ is a finite subset, $\underline{i}, \underline{j} \in I_{F}$ and $k \in[L]$. Let $k_{F}(\underline{i})=k-\sum_{\omega \in F} \nu\left(\omega, i_{\omega}\right)$; then we have

i) $\widehat{e}_{\underline{i}}^{(F, k)}=\left\{\left(k_{F}(\underline{i}), \underline{i}^{\prime}\right) \in X: \underline{i}^{\prime} \in \prod_{\omega \in \Omega}[\eta(\omega)], i_{\omega}^{\prime}=i_{\omega}\right.$ for all $\left.\omega \in F\right\}$.

ii) $\widehat{e}_{\underline{i}, \underline{j}}^{(F, k)}=\left\{\left(\left(k_{F}(\underline{i}), \underline{i}^{\prime}\right),\left(k_{F}(\underline{j}), \underline{\left.\left.j^{\prime}\right)\right):} \begin{array}{l}\underline{i}^{\prime}, \underline{j}^{\prime} \in \prod_{\omega \in \Omega}[\eta(\omega)], i_{\omega}^{\prime}=i_{\omega}, j_{\omega}^{\prime}=j_{\omega} \text { for } \\ \text { all } \omega \in F \text { and } i_{\omega}^{\prime}=j_{\omega}^{\prime} \text { for all } \omega \in \Omega \backslash F\end{array}\right\}\right.\right.$.

Proof. i) follows from the discussion preceding this proposition.

For ii), suppose $(x, y) \in \widehat{e}_{\underline{i}, \underline{j}}^{(F, k)}$. Then $x \in \widehat{e}_{\underline{i}}^{(F, k)}$ and $y \in \widehat{e}_{\underline{j}}^{(F, k)}$. It follows from i) that $k(x)=k_{F}(\underline{i})$ and $k(y)=k_{F}(\underline{j})$. Suppose $\omega \in \Omega \backslash F$. Since $e_{\underline{i}, \underline{j}}^{(F, k)}$ embeds into $\mathfrak{A}_{G}, G=F \cup\{\omega\}$, as $\sum_{s \in[\eta(\omega)]} e_{\underline{i} \sqcup s, \underline{j} \sqcup s}^{(G, k+\nu(\omega, s))}$, we necessarily have $i_{\omega}^{\prime}=j_{\omega}^{\prime}$.

3.1.2 Remarks. 1) Given a finite subset $F \subset \Omega$, let

$$
\begin{aligned}
\widetilde{\mathcal{R}}_{F} & =\left\{(x, y) \in \widetilde{\mathcal{R}}:(x, y) \in \widehat{v} \text { for some matrix unit } v \in \mathfrak{A}_{F}\right\} \\
& =\bigcup\left\{\widehat{e}_{\underline{i}, \underline{j}}^{(F, k)}, \underline{i}, \underline{j} \in I_{F}, k \in[L]\right\} .
\end{aligned}
$$

Then $\widetilde{\mathcal{R}}=\bigcup\left\{\widetilde{\mathcal{R}}_{F}: F\right.$ is a finite subset of $\left.\Omega\right\}$.

2) Let $\pi: X \rightarrow X^{\tau}$ be the dual map of the unital embedding $\iota: \mathcal{D}^{\tau} \hookrightarrow \mathcal{D}$. For $x=(k, \underline{i}) \in X, \pi(x)=\underline{i}$. If $(x, y) \in \widetilde{\mathcal{R}}$ then $(\pi(x), \pi(y)) \in \widetilde{\mathcal{R}}^{\tau}$. On the other hand, if $(\underline{i}, \underline{j}) \in \widetilde{\mathcal{R}}^{\tau}$, we can take $x_{0}=(0, \underline{i})$ and $y_{0}=(\ell, \underline{j})$, where $\ell=$ $\sum_{\omega \in \Omega}\left(\nu\left(\omega, i_{\omega}\right)-\nu\left(\omega, j_{\omega}\right)\right)$. Since $(\underline{i}, \underline{j}) \in \widetilde{\mathcal{R}}^{\tau}, i_{\omega}=j_{\omega}$ for all but finitely many $\omega \in \Omega, \ell$ is well defined and $\left(x_{0}, y_{0}\right) \in \widetilde{\mathcal{R}}$. Clearly, $\left(\pi\left(x_{0}\right), \pi\left(y_{0}\right)\right)=(\underline{i}, \underline{j})$. Suppose $(x, y) \in \widetilde{\mathcal{R}}$ and $(\pi(x), \pi(y))=(\underline{i}, \underline{j})$; then $(x, y)=\left((k, \underline{i}),\left(k^{\prime}, \underline{i}^{\prime}\right)\right)$ for some $k, k^{\prime} \in[L]$ and there exists a finite subset $F$ of $\Omega$ such that $i_{\omega}=j_{\omega}$ for all $\omega \in \Omega \backslash F$ and $k^{\prime}=k+\sum_{\omega \in F}\left(\nu\left(\omega, i_{\omega}\right)-\nu\left(\omega, j_{\omega}\right)\right)=k+\ell$. Therefore, $(x, y)=\left(\tau^{k}\left(x_{0}\right), \tau^{k}\left(y_{0}\right)\right)$.

Furthermore, if $\mathcal{R}_{F}=\widetilde{\mathcal{R}}_{F} \cap \mathcal{R}$, then a point $(x, y) \in \widetilde{\mathcal{R}}_{F}$ belongs to $\mathcal{R}_{F}$ if and only if $\pi(x)$ is less than or equal to $\pi(y)$ in the lexicographic order determined by $\Omega$.

3.1.3 Proposition. $\widetilde{\mathcal{R}}$ is a fiber bundle over $\widetilde{\mathcal{R}}^{\tau}$ with discrete fibers.

Proof. Recall that $X^{\tau}=\prod_{\omega \in \Omega}[\eta(\omega)]$ and

$$
\widetilde{\mathcal{R}}^{\tau}=\left\{\left(i_{\omega}, j_{\omega}\right)_{\omega \in \Omega} \in X^{\tau} \times X^{\tau}: i_{\omega}=j_{\omega} \text { for all but a finite number of } \omega \in \Omega\right\} .
$$

Let $\left(i_{\omega}, j_{\omega}\right)_{\omega \in \Omega} \in \widehat{\mathcal{R}^{\tau}}$. So there exists a finite subset $F$ of $\Omega$ such that $i_{\omega}=j_{\omega}$ for all $\omega \in \Omega \backslash F$. Let $\underline{i}=\left(i_{\omega}\right)_{\omega \in F}$ and $\underline{j}=\left(j_{\omega}\right)_{\omega \in F}$. Then we can define a homeomorphism $\phi:[L] \times \widehat{e}_{\underline{i}, \underline{j}}^{(F)} \rightarrow \pi^{-1}\left(\widehat{e}_{\underline{i}, \underline{j}}^{(\bar{F})}\right)=\bigcup_{k \in[L]} \widehat{e}_{\underline{i}, \underline{j}}^{(F, k)}$ by $\phi((k,(x, y)))=$ $\left(\left(k_{F}(\underline{i}), x\right),\left(k_{F}(\underline{j}), y\right)\right)$.

Viewing $\widetilde{\mathcal{R}}$ as a relation on $X$, one can ask if local cross-sections $\sigma: U \subset X^{\tau} \rightarrow X$ can be found which preserve the equivalence relation. The answer is negative, if $\mathfrak{A}$ is simple and $L>1$. 
3.1.4 Proposition. If $\mathfrak{A}$ is simple and $L>1$, then there is no local cross-section $\sigma: U \subset X^{\tau} \rightarrow X$ which preserves equivalence classes.

Proof. Suppose there is a nonempty clopen set $U \subset X^{\tau}$ and a cross-section $\sigma: U \rightarrow$ $X$ such that for any $x, y \in U$, if $(x, y) \in \widetilde{\mathcal{R}}^{\tau}$, then $(\sigma(x), \sigma(y)) \in \widetilde{\mathcal{R}}$. Now any cross section $\sigma$ maps $U$ homeomorphically onto $\sigma(U)\left[\mathrm{Hu}\right.$, p. 70]. As $X^{\tau}$ is compact and $U$ closed, $U, \sigma(U)$ are compact. Set $\sigma_{k}=\tau^{k} \circ \sigma, 0 \leq k<L$, and $U_{k}=\sigma_{k}(U)$.

Let $u \in U$. Then $\sigma(u)=(\ell(u), u)$ for some $\ell(u) \in[L]$ and $\tau^{k} \circ \sigma(u)=$ $(k+\ell(u), u)$. Thus for $0 \leq k, \ell<L$ and $u, v \in U, \tau^{k} \circ \sigma(u)=\tau^{\ell} \circ \sigma(v)$ iff $u=v$ and $k=\ell$. Hence, the $U_{k}, 0 \leq k<L$, are pairwise disjoint. It follows that the sets $U_{k}, k \in[L]$, form a clopen partition of $\pi^{-1}(U)$.

As $\mathfrak{A}$ is a simple AF algebra, the orbit $\mathcal{O}(x)=\{y \in X:(x, y) \in \widetilde{\mathcal{R}}\}$ of any point $x$ is dense in $X$. Thus, given any $x=(k, \underline{i}) \in U_{0}$ there is a $y=\left(k^{\prime}, j\right) \in U_{1}$ with $(x, y) \in \widetilde{\mathcal{R}}$. Hence, there exists a finite subset $F$ such that

$$
i_{\omega}=j_{\omega} \text { for all } \omega \in \Omega \backslash F \quad \text { and } \quad k+\sum_{\omega \in F} \nu\left(\omega, i_{\omega}\right)=k^{\prime}+\sum_{\omega \in F} \nu\left(\omega, j_{\omega}\right) .
$$

Then $\left(\pi(x), \pi \circ \tau^{-1}(y)\right) \in \widetilde{\mathcal{R}}^{\tau}$. By assumption,

$$
\left(x, \tau^{-1}(y)\right)=\left(\sigma \circ \pi(x), \sigma \circ \pi \circ \tau^{-1}(y)\right) \in \widetilde{\mathcal{R}} .
$$

Since $\tau^{-1}(y)=\left(k^{\prime}-1, \underline{j}\right)$, there is a finite subset $G$ of $\Omega$ such that

$$
i_{\omega}=j_{\omega} \text { for all } \omega \in \Omega \backslash G \quad \text { and } \quad k+\sum_{\omega \in G} \nu\left(\omega, i_{\omega}\right)=k^{\prime}+\sum_{\omega \in G} \nu\left(\omega, j_{\omega}\right)-1 .
$$

This gives

$$
k^{\prime}+\sum_{\omega \in F \cup G} \nu\left(\omega, j_{\omega}\right)=k+\sum_{\omega \in F \cup G} \nu\left(\omega, i_{\omega}\right)=k^{\prime}+\sum_{\omega \in F \cup G} \nu\left(\omega, j_{\omega}\right)-1,
$$

a contradiction. Thus, no such cross-section exists.

3.2 Minimal points in $X$. The semigroupoid $\mathcal{R}$ defines a partial order on $X$ : $x \prec y$ if $(x, y) \in \mathcal{R}$. $x$ is said to be a minimal (resp., maximal) point if it is minimal (resp., maximal) in this ordering. In case $L=1$, or more generally in case the TAF algebra $\mathcal{T}$ is the closed union of an increasing sequence $\left(\mathcal{T}_{n}\right)_{n=1}^{\infty}$ of finite dimensional subalgebras such that $\mathcal{T}_{n}+\mathcal{T}_{n}^{*}$ is a factor, there is a unique minimal point in $X$, the maximal ideal space of the diagonal. Namely,

$$
\left\{x_{\min }\right\}=\bigcap_{n=1}^{\infty} \widehat{e}_{\min }^{(n)}
$$

where $e_{\min }^{(n)}$ is the unique matrix unit in the diagonal $\mathcal{D}_{n}$ of $\mathcal{T}_{n}$, minimal with respect to the diagonal ordering [PPW1, p. 98].

If the TAF algebra $\mathcal{T}=\mathcal{T}(\Omega, \Phi)$, then $\mathcal{T}^{\tau}$ satisfies the above condition, so that $X^{\tau}$ has a unique minimal point, which is clearly the point

$$
\underline{0}=\left(0_{\omega}\right)_{\omega \in \Omega} \in \prod_{\omega \in \Omega}[\eta(\omega)] .
$$

Assume now that the embeddings $\Phi_{F, G}^{\nu}$ are in standard form (cf. 2.5), which means $\nu(\omega, 0)=0, \omega \in \Omega$. For any finite subset $F \subset \Omega$, let $e^{(F, k)}$ denote the central projection in $\mathfrak{A}_{F}$ onto the factor $\mathfrak{A}_{F, k}$ (as in 1.2). Define $\left\{x_{k}\right\}=\pi^{-1}(\underline{0}) \cap \widehat{e}^{(F, k)}$. 
3.2.1 Proposition. $\left\{x_{0}, \ldots, x_{L-1}\right\}$ is the set of minimal points of $X$. Also, the definition of $x_{k}$ is independent of $F \subset \Omega$.

Proof. If $y \in X$ is a minimal point, by Remark 3.1.2 2), $\pi(y)$ is minimal in $X^{\tau}$; hence $\pi(y)=\underline{0}$. On the other hand, by the same remark, the points $x_{k} \in \pi^{-1}(\underline{0})$ are minimal in $X$.

To see that the definition of $x_{k}$ is independent of the finite subset $F \subset \Omega$, let $e_{\min }^{(F, k)}=e_{(0, \ldots, 0)}^{(F, k)}$. The standard form of the embeddings $\Phi^{\nu}$ implies that, if $F \subset G \subset \Omega, \Phi_{F, G}^{\nu}$ maps $e_{\min }^{(F, k)}$ to $e_{\min }^{(G, k)}+$ other diagonal matrix units. In other words, $\left\{x_{k}\right\}=\bigcap_{\substack{F \subset \Omega \\ F \text { finite }}} \widehat{e}_{\min }^{(F, k)}$.

3.2.2 Remark. The above proposition shows that the ordering of the factors $\mathfrak{A}_{F, k}$ of $\mathfrak{A}_{F}$ is not 'arbitrary'.

The general question of when two TAF algebras $\mathcal{T}(\Omega, \Phi), \mathcal{T}\left(\Omega^{\prime}, \Phi^{\prime}\right)$ are isomorphic, to which Power has given a complete answer in case $L=1$, is evidently more delicate for the larger class under consideration. However, one criterion for isomorphism is easy to state.

3.2.3 Proposition. $\mathcal{T}(\Omega, \Phi,) \cong \mathcal{T}\left(\Omega^{\prime}, \Phi^{\prime},\right)$ implies $L(\Phi)=L\left(\Phi^{\prime}\right)$.

Proof. Isomorphism of the TAF algebras implies isomorphism of the corresponding semigroupoids, so they must have the same number of minimal points.

3.3 Locally order-preserving embeddings. Let $\mathcal{T}$ be a TAF algebra. Recall that the diagonal ordering [PPW1] on the diagonal projections is a partial order defined as follows: for $p, q$ projections in $\mathcal{D}=\mathcal{T} \cap \mathcal{T}^{*}, p \prec q$ if there is a partial isometry $w \in \mathcal{N}_{\mathcal{D}}(\mathcal{T})$ (the normalizer of $\mathcal{D}$ in $\mathcal{T}$ ) with $w w^{*}=p, w^{*} w=q$. Each element $v \in \mathcal{N}_{\mathcal{D}}(\mathcal{T})$ defines a map with domain $\left\{p \in \mathcal{P}(\mathcal{D}): p \leq v v^{*}\right\}$ and range $\left\{p \in \mathcal{P}(\mathcal{D}): p \leq v^{*} v\right\}$ by $p \rightarrow v^{*} p v$. An element $v$ of the normalizer is said to be order-preserving if for any two elements $p, q \in \mathcal{P}(\mathcal{D})$ satisfying $p, q \leq v v^{*}$ with $p \prec q$, then $v^{*} p v \prec v^{*} q v$. Let $\mathcal{N}_{\mathcal{D}}^{o p}(\mathcal{T})$ denote the set of all $v \in \mathcal{N}_{\mathcal{D}}(\mathcal{T})$ with this property.

If $\mathcal{S}, \mathcal{T}$ are finite dimensional TAF algebras and $\Phi: \mathcal{S} \rightarrow \mathcal{T}$ is a regular embedding (i.e., $\varphi$ in $*$-extendible and maps matrix units in $\mathcal{S}$ to sum of matrix units on $\mathcal{T}$ ), $\varphi$ is said to be locally order preserving if $\varphi(e)$ is in the order-preserving normalizer of $\mathcal{T}$, for every matrix unit $e \in \mathcal{S}$. Suppose now that $\mathcal{T}$ is a TAF algebra of the form $\mathcal{T}(\Omega, \Phi)$. It is easy to see that the embeddings $\Phi_{F, G}: \mathcal{T}_{F} \hookrightarrow \mathcal{T}_{G}$ are locally order preserving. To see if an element of the normalizer of $\mathcal{T}_{G}$ is order-preserving, it is enough to check it on diagonal matrix units. Since any two related matrix units lie in the same factor of $\mathcal{T}_{G}$, we need only look at $e^{(G, k)} \Phi_{F, G}(e), e=e_{\underline{i}, \underline{j}}^{(F, l)}$ a matrix unit of $\mathcal{T}_{F}$. This has the form $v=\sum_{t \in I} e_{\left(i_{1}, \ldots, i_{r}, t, \ldots, i_{m}\right)\left(j_{1}, \ldots, j_{r}, t, \ldots, j_{m}\right)}^{(G, k)}$, for $\underline{i}=\left(i_{1}, \ldots, i_{m}\right), \underline{j}=\left(j_{1}, \ldots, j_{m}\right)$, and $G=F \cup\{\omega\}, \omega_{r}<\omega<\omega_{r+1}$, and $I$ is some subset of $[\eta(\omega)]$. If $e_{1}, e_{2} \in \mathcal{D}_{G}$ satisfy $e_{1}, e_{2} \leq v v^{*}$, then $e_{1}=e_{\left(i_{1}, \ldots, i_{r}, t_{1}, \ldots, i_{m}\right)}^{(G, k)}, e_{2}=$

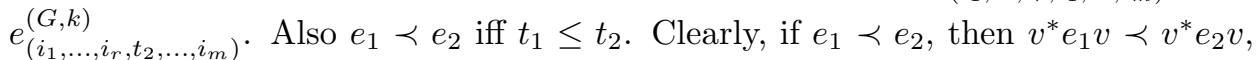
as $v^{*} e_{1} v=e_{\left(j_{1}, \ldots, j_{r}, t_{1}, \ldots, j_{m}\right)}^{(G, k)}, v^{*} e_{2} v=e_{\left(j_{1}, \ldots, j_{r}, t_{2}, \ldots, j_{m}\right)}^{(G, k)}$. In fact the same calculation will work if $G \backslash F$ is any finite set, so that all the embeddings $\Phi_{F, G}$ are locally order-preserving. 
3.3.1 Proposition. For $=\mathcal{T}(\Omega, \Phi)$ we have

1) The closed span of $\mathcal{N}_{\mathcal{D}}^{o p}(\mathcal{T})$ is $\mathcal{T}$.

2) $\mathcal{R}=\bigcup\left\{\widehat{v}: v \in \mathcal{N}_{\mathcal{D}}^{o p}(\mathcal{T})\right\}$.

Proof. These follow from [DH, Theorem 18].

Suppose $\mathcal{T}=\underline{\lim }\left(\mathcal{T}_{n}, \varphi_{n}\right)$, where $\mathcal{T}_{n} \cong T_{k_{n}}$, the upper triangular subalgebra of $M_{k_{n}}$, and the embeddings $\varphi_{n}$ are lexicographic. This is a special case of the situation considered above, and so the embeddings $\varphi_{n}$, as well as the compositions $\varphi_{n, m}=\varphi_{m} \circ \varphi_{m-1} \circ \cdots \circ \varphi_{n}$ for $m>n$, are locally order-preserving. (This is also immediate from [DH, Theorem 8].) One can ask, conversely, if every such inductive limit in which finite compositions are locally ordering-preserving must be lexicographic. The following example, due to Donsig, shows this is not the case.

3.3.2 Example. Define $\varphi_{n}: M_{3 \cdot 2^{n}} \rightarrow M_{3.2^{n+1}}$ by

$$
\varphi_{n}\left[\begin{array}{ccc}
A & & \\
& B & \\
& & C
\end{array}\right]=\left[\begin{array}{llllll}
A & & & & & \\
& B & & & & \\
& & A & & & \\
& & & B & & \\
& & & & C & \\
& & & & & C
\end{array}\right]
$$

on diagonal blocks (i.e. $A, B, C$ are $2^{n} \times 2^{n}$ blocks) and extend uniquely to a locally order-preserving map $T_{2 \cdot 3^{n}} \rightarrow T_{2 \cdot 3^{n}}$, and by *-extendibility, to $M_{2 \cdot 3^{n}} \rightarrow M_{2 \cdot 3^{n}}$.

Finite compositions of successive $\varphi_{n}$ 's are locally order-preserving, but $\varphi_{n}$ is not lexicographic.

3.4 Subsets of $\Omega$ and their corresponding subalgebras. Let $(\Omega, \Phi)$ be a lexicographic inductive system, let $\Omega^{\prime}$ be an infinite subset of $\Omega$, and for $F \subset G$ finite subsets of $\Omega^{\prime}, \Phi_{F, G}^{\prime}=\Phi_{F, G}: \mathfrak{A}_{F} \hookrightarrow \mathfrak{A}_{G}$ a $C^{*}$-embedding. Then $\left(\Omega^{\prime}, \Phi^{\prime}\right)$ is an inductive system, and one can form the inductive limit $\mathfrak{A}^{\prime}=\lim _{F \subset G \subset \Omega^{\prime}}\left(\mathfrak{A}_{F}, \Phi_{F, G}^{\prime}\right)$. By fundamental properties of inductive limits, there is a unique embedding $\Phi_{\Omega^{\prime}}$ : $\mathfrak{A}^{\prime} \rightarrow \mathfrak{A}$ with the following property: if $F \subset \Omega^{\prime}$ is any finite subset, let $\Phi_{F}^{\prime}$, resp. $\Phi_{F}$, denote the canonical embeddings $\mathfrak{A}_{F} \hookrightarrow \mathfrak{A}^{\prime}$, resp., $\mathfrak{A}_{F} \hookrightarrow \mathfrak{A}$. Then the diagram

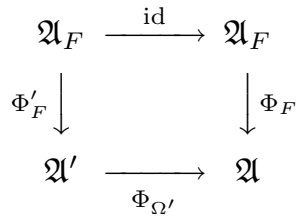

commutes.

One has similar statements for the respective triangular subalgebras $\mathcal{T}_{F}, \mathcal{T}^{\prime}, \mathcal{T}$, as well as for the diagonal subalgebras $\mathcal{D}_{F}, \mathcal{D}^{\prime}, \mathcal{D}$.

In particular, the embedding $\mathcal{D}^{\prime}=\mathcal{D}\left(\Omega^{\prime}, \Phi\right)$ into $\mathcal{D}=\mathcal{D}(\Omega, \Phi)$ yields, by transposition, a continuous surjection $\zeta$ from the maximal ideal space $X$ of $\mathcal{D}$ onto the maximal ideal space $X^{\prime}$ of $\mathcal{D}^{\prime}$. Furthermore, $\zeta \times\left.\zeta\right|_{\widetilde{\mathcal{R}}}$ maps the groupoid $\widetilde{\mathcal{R}}$ of $\mathfrak{A}$ onto the groupoid $\widetilde{\mathcal{R}}^{\prime}$ of $\mathfrak{A}^{\prime}$.

3.4.1 Lemma. $\zeta \times\left.\zeta\right|_{\widetilde{\mathcal{R}}}: \widetilde{\mathcal{R}} \rightarrow \widetilde{\mathcal{R}}^{\prime}$ is a continuous surjection. 
Proof. Let $(x, y) \in \widetilde{\mathcal{R}}$, and $U$ a neighborhood of $\left(x^{\prime}, y^{\prime}\right)=(\zeta(x), \zeta(y)) \in \widetilde{\mathcal{R}}^{\prime}$. Since the sets $\widehat{e}_{\underline{i}, j}^{(F, k)}\left(F \subset \Omega^{\prime}\right)$ form a basis for the topology of $\widetilde{\mathcal{R}}^{\prime}$, we may assume $U=$ $\widehat{e}_{\underline{i}, \underline{j}}^{(F, k)} \subset \widetilde{\mathcal{R}}^{\prime}$. We need a neighborhood $V$ of $(x, y)$ such that $(\zeta \times \zeta)(V) \subset U$. Take $V=\widehat{e}_{\underline{i}, \underline{j}}^{(F, k)} \subset \widetilde{\mathcal{R}}$.

3.4.2 Remark. A similar statement holds if $\widetilde{\mathcal{R}}, \widetilde{\mathcal{R}}^{\prime}$ are replaced by $\mathcal{R}, \mathcal{R}^{\prime}$.

In the case $L=1$, the map $\zeta: X \rightarrow X^{\prime}$ can be interpreted as $\zeta\left(\left(x_{\omega}\right)_{\omega \in \Omega}\right)=$ $\left(x_{\omega}\right)_{\omega \in \Omega^{\prime}}$, deleting those coordinates $\omega \in \Omega \backslash \Omega^{\prime}$. Let $X_{0}$ be the subset of $X$ consisting of those points $\left(x_{\omega}\right)_{\omega \in \Omega}$ for which $x_{\omega}=0$ for $\omega \in \Omega \backslash \Omega^{\prime}$. Identifying $X^{\prime}$ with $X_{0}$, we can also identify $\widetilde{\mathcal{R}}^{\prime}$ with $\widetilde{\mathcal{R}} \cap\left(X_{0} \times X_{0}\right)$.

3.4.3 Proposition. Let $\Omega^{\prime}$ be an infinite subset of $\Omega$. If $(\Omega, \Phi)$ is an inductive system with $L=1$, then $\widetilde{\mathcal{R}}^{\prime}$ (resp. $\mathcal{R}^{\prime}$ ) can be identified with a subset of $\widetilde{\mathcal{R}}$ (resp., $\mathcal{R})$ as indicated above.

\section{LAT $\mathcal{T}(\Omega, \Phi)$}

While it is known that $\operatorname{Lat}(\mathcal{T})$ is a nest for the case when $\mathcal{T}$ a is maximal TAF and the ambient AF algebra is UHF (e.g., Proposition 2.1 of [Pe Wa]), no analogue has been stated for simple AF algebras.

4.1 Proposition. Let $\mathfrak{A}=\overline{\cup \mathfrak{A}_{n}}$ be a simple (unital) AF algebra, and $\mathcal{T}_{n} \subset \mathfrak{A}_{n}$ a maximal triangular subalgebra. Assume that the embeddings $\mathfrak{A}_{n} \hookrightarrow \mathfrak{A}_{n+1}$ are regular with respect to the masa $\mathcal{D}_{n}=\mathcal{T}_{n} \cap \mathcal{T}_{n}^{*}$ and map $\mathcal{T}_{n} \hookrightarrow \mathcal{T}_{n+1}$. Let $\mathcal{T}=\overline{\cup \mathcal{T}_{n}}$. Then $\operatorname{Lat}(\mathcal{T})$ is a nest.

Proof. Suppose not; then there are projections $p_{1}, p_{2} \in \operatorname{Lat}(\mathcal{T})$ with $p_{1}{ }^{\perp} p_{2}, p_{1} p_{2}{ }^{\perp}$ both nonzero. Since any projection in $\operatorname{Lat}(\mathcal{T})$ commutes with $\mathcal{D}=\overline{\cup \mathcal{D}_{n}}$, and $\mathcal{D}$ is a canonical masa, it follows that $\operatorname{Lat}(\mathcal{T}) \subset \mathcal{D}$ and hence $p_{1}, p_{2} \in \mathfrak{A}_{n}$ for some $n$. As $\mathfrak{A}$ is a simple AF-algebra, there is an $m>n$ such that each of $p_{1}{ }^{\perp} p_{2}, p_{1} p_{2}{ }^{\perp}$ partially embeds in the same factor of $\mathfrak{A}_{m}$. Thus there is a factor $\mathfrak{A}_{m, k} \subset \mathfrak{A}_{m}$ and a central projection $e^{(m, k)}$ onto $\mathfrak{A}_{m, k}$ with $p_{1}{ }^{\perp} p_{2} e^{(m, k)} \neq 0$ and $p_{1} p_{2} \perp^{(m, k)} \neq 0$. Let $I_{m, k}$ index the matrix units in $\mathfrak{A}_{m, k}$, so that $i \leq j$ iff $e_{i}^{(m, k)}<e_{j}^{(m, k)}$ in the diagonal ordering. Since $p_{l} \in$ Lat $\mathcal{T}, l=1,2$, there are integers $r_{l}$ such that $e_{i}^{(m, k)} \leq p_{l}$ for $i \in I_{m, k}, i \leq r_{l}$, and $e_{j}^{(m, k)} \perp p_{l}$ for $j \in I_{m, k}, j>r_{l}, l=1,2$. But then $p_{1}{ }^{\perp} p_{2} e^{(m, k)}=0$ if $r_{1} \geq r_{2}$, while $p_{1} p_{2}{ }^{\perp} e^{(m, k)}=0$ if $r_{1} \leq r_{2}$. Thus Lat $(\mathcal{T})$ is a nest.

4.2 Lemma. Suppose $L=1$. Express $\Omega=\Omega_{0}+\Omega_{1}$ (order-sum), where $\Omega_{0}$ is the maximal well-ordered initial segment which contains no limit ordinal. (Hence $\Omega_{0}$ either is finite or else has order type $\mathbb{Z}^{+}$.) Then $\mathcal{L}=\operatorname{Lat} \mathcal{T}(\Omega, \Phi)=\{0\} \cup\left\{P_{\underline{i}}^{(F)}\right.$ : $\left.F \subset \Omega_{0}, \underline{i} \in I_{F}\right\}$, where $P_{\underline{i}}^{(F)}=\sum_{\underline{j} \leq i} e_{\underline{j}}^{(F)}$.

Proof. That $P_{\underline{i}}^{(F)} \in \mathcal{L}$ is a straightforward observation. Suppose $p \in \operatorname{Lat} \mathcal{T}$ is not of the form $P_{\underline{i}}^{(F)}\left(F \subset \Omega_{0}, \underline{i} \in I_{F}\right)$. Since Lat $\mathcal{T} \subset \mathcal{D}$, necessarily $p \in \mathcal{D}_{F}$ for some finite $F \subset \Omega$. Since the upper triangular matrix units $e_{\underline{i}, \underline{j}}^{(F)}, \underline{i} \leq \underline{j}$, span $\mathcal{T}_{F}$, and as any element of Lat $\mathcal{T} \cap \mathcal{D}_{F}$ also lies in Lat $\mathcal{T}_{F}, p$ has the form $p=$ 
$\sum_{\underline{i} \underline{i}_{0}} e_{\underline{i}}^{(F)}$ for some index $\underline{i}_{0} \in I_{F}$. Now if $F \not \subset \Omega_{0}$, say $\omega \in \Omega_{1} \cap F$, then there is an $\omega^{\prime} \in \Omega \backslash F$ with $\omega^{\prime}<\omega$. One verifies that for $G=F \cup\left\{\omega^{\prime}\right\}, \Phi_{F, G}(p) \notin$ Lat $\mathcal{T}_{G}$. Thus, $p \notin$ Lat $\mathcal{T}$.

4.3 Notation. For an inductive system $(\Omega, \Phi)$ there is a conditional expectation $E^{\tau}: \mathfrak{A} \rightarrow \mathfrak{A}^{\tau}$ defined by

$$
E^{\tau}(a)=\frac{1}{L} \sum_{k=0}^{L-1} \tau^{k}(a)
$$

Observe that $E^{\tau}$ also maps $\mathcal{T}$ onto $\mathcal{T}^{\tau}$, and $\mathcal{D}$ onto $\mathcal{D}^{\tau}$.

4.4 Proposition. Let $(\Omega, \Phi)$ be an inductive system such that $\mathfrak{A}(\Omega, \Phi)$ is a simple AF-algebra. Set $\mathcal{L}=\operatorname{Lat}(\mathcal{T}(\Omega, \Phi))$ and $\mathcal{L}^{\tau}=\operatorname{Lat}\left(\mathcal{T}^{\tau}(\Omega, \Phi)\right)$. Then

a) $\mathcal{L}$ is a nest;

b) $E^{\tau}(\mathcal{L})=\mathcal{L}^{\tau}$

c) $\mathcal{L}=\left\{p: \exists q \in \mathcal{L}^{\tau}\right.$ with $p=\bigvee\left\{p^{\prime}: p^{\prime}\right.$ is a projection in $\left.\left.\mathcal{D}, E^{\tau}\left(p^{\prime}\right)=q\right\}\right\}$

Proof. a) follows from Proposition 4.1.

b) Clearly $E^{\tau}(\mathcal{L}) \subseteq \mathcal{L}^{\tau}$. Let $q \in \mathcal{L}^{\tau}$. By Lemma 4.2 there is a finite subset $F \subset \Omega_{0}$ such that $q=P_{\underline{i}}^{(F)}$ for some $\underline{i} \in I_{F}$. Let $p=\sum_{k \in[L]} \sum_{\substack{j \leq \underline{i} \\ j \\ \underline{j} \in I_{F}}} e_{j}^{(F, k)}$. It is obvious that $p \in \operatorname{Lat}\left(\mathcal{T}_{F}\right)$. To see $p \in \mathcal{L}=\operatorname{Lat} \mathcal{T}$, it is enough to show that if $G$ is any finite subset of $\Omega$ containing $F$, then $\Phi_{F, G}(p) \in$ Lat $\mathcal{T}_{G}$. Now $F$ can be taken to be an initial segment of $\Omega_{0}$; i.e. $F=\left\{\omega_{1}, \ldots, \omega_{n}\right\}$, where $\omega_{j}$ is the $j^{\text {th }}$ element of $\Omega, 1 \leq j \leq n$. If $\omega \in \Omega \backslash F$ and $G=F \cup\{\omega\}$, then

$$
\Phi_{F, G}\left(\sum_{k \in[L]} e_{\underline{j}}^{(F, k)}\right)=\sum_{s \in[\eta(\omega)]} \sum_{k \in[L]} e_{\underline{j} \sqcup s}^{(G, k+\nu(\omega, s))} .
$$

As $k+\nu(\omega, s)$ is a sum mod $L, \sum_{k \in[L]} e_{\underline{j}\lrcorner s}^{(G, k+\nu(\omega, s))}=\sum_{k \in[L]} e_{\underline{j} \sqcup s s}^{(G, k)}$. Note that as $\omega_{n}<\omega, \underline{j} \sqcup s=\left(j_{1}, \ldots, j_{n}, s\right)\left(\underline{j}=\left(j_{1}, \ldots, j_{n}\right)\right)$. Set $\underline{j}_{0}=\left(j_{1}, \ldots, j_{n}, \eta(\omega)-\right.$ 1). Then $\Phi_{F, G}\left(\sum_{k \in[L]} e_{\underline{j}}^{(F, k)}\right)$ can be expressed as $\sum_{k \in[L]} \sum_{\substack{j^{\prime} \leq j_{0} \\ j^{\prime} \in I_{G}}} e_{\underline{j}^{\prime}}^{(G, k)}$. Thus $\Phi_{F, G}(p)=\sum_{k \in[L]} \sum_{\substack{i \leq i_{0} \\ \underline{i} \in I_{G}}} e_{\underline{i}}^{(G, k)}$, where $\underline{i}_{0}$ is defined analogously. Again, this is obviously in Lat $\mathcal{T}_{G}$. If $G \backslash F$ is any finite subset of $\Omega$, we can repeat the above argument to show $\Phi_{F, G}(p) \in$ Lat $\mathcal{T}_{G}$. Hence $p \in \mathcal{L}$. Clearly $E^{\tau}(p)=q$.

c) Let $q \in \mathcal{L}^{\tau}$. Then the $p$ constructed in b) has the property $p=\bigvee\left\{p^{\prime}: p^{\prime}\right.$ a projection in $\left.\mathcal{D}, E^{\tau}\left(p^{\prime}\right)=q\right\}$. Indeed, if $p^{\prime}$ were any projection in $\mathcal{D}$ with $p^{\prime} \not \leq p$, then viewing $p, p^{\prime}$ as elements of some finite dimensional subalgebra $\mathfrak{A}_{G}, p$ has the form $\sum_{k \in[L]} \sum_{\substack{i \leq i_{0} \\ i \in \underline{j}_{G}}} e_{\underline{i}}^{(G, k)}$, so $p^{\prime}$ necessarily contains as a summand some diagonal matrix $e_{\underline{i}}^{(G, k)}$ with $\underline{i} \in I_{G}, \underline{i}>\underline{i}_{0}$. But then $E^{\tau}\left(p^{\prime}\right) \neq q$.

It remains to show there are no other projections in Lat $\mathcal{T}$. If $p \in$ Lat $\mathcal{T}$, then $p \in \mathfrak{A}_{F}$, for some finite $F \subset \Omega$. By b), $E^{\tau}(p) \in \mathcal{L}^{\tau}$, so $E^{\tau}(p)=q_{1} \in$ 
Lat $\mathcal{T}^{\tau} \cap \mathfrak{A}_{F}^{\tau}$. Now $E^{\tau}$ is faithful, so $p \neq 0$ implies $q_{1} \neq 0$. Let $q_{2}$ be the immediate predecessor of $q_{1}$ in Lat $\mathcal{T}^{\tau} \cap \mathfrak{A}_{F}^{\tau}$. Set $p_{l}=\bigvee\left\{p^{\prime}: p^{\prime}\right.$ is a projection in $\left.\mathcal{D}_{F}, E^{\tau}\left(p^{\prime}\right)=q_{l}\right\}, l=1,2$. We know from the previous paragraph that $p_{l} \in$ Lat $\mathcal{T}$. Since Lat $\mathcal{T}$ is a nest, $p_{2} \leq p \leq p_{1}$, and $p$ has the form $p=$ $p_{2}+\sum_{s} e_{\underline{i}_{1}}^{\left(F, k_{s}\right)}$, where $p_{1}=P_{\underline{i}_{1}}^{(F)}, p_{2}=P_{\underline{i}_{2}}^{(F)}$ (notation as in Lemma 4.2), and the integers $k_{s}$ range over some subset $S$ of $[L]$. Now if $p \neq p_{1}, p_{2}$, then $S$ is a nonempty proper subset of $[L]$. So there is a $k_{0} \in[L]$ with $k_{s} \neq k_{0}$ for all $s$ in the sum. For convenience, assume that the embeddings $\Phi_{F, G}$ are in standard form (cf. Remark 2.5). Since $\mathfrak{A}$ is a simple AF algebra, there is a finite subset $G \supset F$ such that each factor of $\mathfrak{A}_{F}$ partially embeds in each factor of $\mathfrak{A}_{G}$. We may also assume, enlarging $G$ if necessary, that for some $\omega \in G \backslash F, \omega^{\prime}<\omega$ for $\omega^{\prime} \in F$. Let $m=\operatorname{card}(G \backslash F)$. Now $\Phi_{F, G}(p)$ does not contain $e_{\underline{i_{1}} \underline{ } \underline{\underline{0}}}^{\left(G, k_{0}\right)}$, but does contain $e_{\underline{i_{1}} \underline{\underline{L}} \underline{\underline{s}}}^{\left(G, k_{0}\right)}$ as a summand, for some $m$-tuple $\underline{s}=\left(s_{1}, \ldots, s_{m}\right)$. Furthermore, the $(n+m)$-tuple $\underline{i}_{1} \sqcup \underline{s}$ denotes the coordinates of $\underline{i}_{1}$ and $\underline{s}$ re-ordered according to the $\Omega$-ordering, and the last coordinate will not come from $\underline{i}_{1}$. Thus, $\Phi_{F, G}(p) \cdot e^{\left(G, k_{0}\right)}$ is not an element of $\operatorname{Lat}\left(\mathcal{T}_{G, k_{0}}\right)$, as any such element has the form $\sum_{j \leq \underline{i}} e^{\left(G, k_{0}\right)}$ for some $\underline{i} \in I_{G}$. Hence $\Phi_{F, G}(p) \notin$ Lat $\mathcal{T}_{G}$, which implies $p \notin$ Lat $\mathcal{T}$.

4.5 Remarks. 1) If $\mathfrak{A}$ is not simple, Lat $\mathcal{T}$ may contain projections in addition to those described in the proposition.

2) Regarding $\mathfrak{A}^{\tau} \subseteq \mathfrak{A}$, then from the construction of the projection in $\operatorname{Lat}(\mathcal{T})$ in Proposition $4.4 \mathrm{~b}$ ), we see that $E^{\tau}(p)=p$ for $p \in \operatorname{Lat}(\mathcal{T})$. So $\operatorname{Lat}(\mathcal{T})$ and $\operatorname{Lat}\left(\mathcal{T}^{\tau}\right)$ coincide.

3) The nest $\operatorname{Lat}(\mathcal{T})$ is maximal. This follows from Proposition $4.4 \mathrm{c}$ ).

4.6 Corollary. Let $(\Omega, \Phi)$ be an inductive system such that $\mathfrak{A}(\Omega, \Phi)$ is a simple AF algebra. Then $\mathcal{T}(\Omega, \Phi)$ is a nest algebra if and only if $\Omega$ has order type $\mathbb{Z}^{+}$.

Proof. Given the description of $\mathcal{L}=$ Lat $\mathcal{T}$ in the proposition, it is easy to see that if $\Omega=\mathbb{Z}^{+}$, a matrix unit $v$ belongs to $\operatorname{Alg} \mathcal{L}$ if and only if it is upper triangular.

On the other hand, if $\Omega$ is not order isomorphic to $\mathbb{Z}^{+}$, then the subset $\Omega_{1}$ in the decomposition $\Omega=\Omega_{0}+\Omega_{1}$ is nonvoid. If $v$ is any matrix unit in $\mathfrak{A}_{F}$ for $F \subset \Omega_{1}$, and $p \in \operatorname{Lat} \mathcal{T}$, then $p v=v p$. Thus $\mathfrak{A}_{F} \subset \operatorname{Alg} \mathcal{L}$, and $\operatorname{Alg} \mathcal{L}$ is not triangular.

4.7 Remarks. 1) If $\Omega=\mathbb{Z}^{+}$and $L=1, \mathcal{T}(\Omega, \Phi)$ is the refinement algebra; in particular, $C^{*}(\mathcal{L})=\mathcal{D}$. If $L>1$, then $\mathcal{L}$ does not generate the diagonal. It is not difficult to show that $C^{*}(\mathcal{L})$ is a subalgebra of $\mathcal{D}$ of codimension $L$. In fact, if $e^{(0)}, \ldots, e^{(L-1)}$ are the central projections onto the factors of any $\mathfrak{A}_{F}$, then $\mathcal{D}$ is generated by $\left(\mathcal{L}, e^{(0)}, \ldots, e^{(L-1)}\right)$.

2) If $\Omega=\mathbb{Z}^{+}$and $L>1$ (and $\mathfrak{A}$ is simple), the nest $\mathcal{L}=$ Lat $\mathcal{T}$ is not a nest of uniform multiplicity in the sense of [Pe Wa, Definition 2.27]. This is because the only $\mathcal{D}$-normalizing partial isometries commuting with $\mathcal{L}$ are in $\mathcal{D}$.

3) Note, in particular, that if $\Omega$ is $\mathbb{Z}^{+} \cup\{\infty\}$, then $\mathcal{T}(\Omega, \Phi)$ is not a nest algebra.

\section{AnALYTiCITY}

Let $\mathcal{T}$ be a TAF subalgebra of (unital) AF algebra $\mathfrak{A}$ with diagonal $\mathcal{D}=\mathcal{T} \cap \mathcal{T}^{*}$, $\widetilde{\mathcal{R}}$ the groupoid corresponding to the pair $(\mathfrak{A}, \mathcal{D})$, and $\mathcal{R}$ the semigroupoid defined 
by $\mathcal{T}$. A 1-cocycle is a continuous function $d: \widetilde{\mathcal{R}} \rightarrow \mathbb{R}$ with the property that $d(x, y)+d(y, z)=d(x, z)$ (cocycle condition) for all $(x, y),(y, z) \in \widetilde{\mathcal{R}}$. $d$ is said to be a cocycle for $\mathcal{T}$ if, in addition, $d(x, y) \geq 0$ for all $(x, y) \in \mathcal{R}$, and $d(x, y)=0$ if and only if $y=x$. A TAF algebra $\mathcal{T}$ is said to be analytic if there is some cocycle $d$ for $\mathcal{T}$.

5.1 Lemma. Let $(\Omega, \Phi)$ be an inductive system. $\mathcal{T}(\Omega, \Phi)$ is analytic if and only if the $\tau$-fixed subalgebra $\mathcal{T}^{\tau}$ is analytic.

Proof. Suppose $d: \widetilde{\mathcal{R}} \rightarrow \mathbb{R}$ is a cocycle for $\mathcal{T}(\Omega, \Phi)$, i.e., $d(x, y) \geq 0$ if and only if $(x, y) \in \mathcal{R}$. Let $(x, y)=(\underline{i}, \underline{j}) \in \widetilde{\mathcal{R}}^{\tau}$. Then there exists a finite subset $F \subset \Omega$ such that $i_{\omega}=j_{\omega}$ for all $\omega \in \Omega \backslash F$. Let $x_{0}=(0, x), y_{0}=(\ell, y)$, where $\ell=\sum_{\omega \in F}\left(i_{\omega}-j_{\omega}\right)$. Define $x_{k}=\tau^{k}\left(x_{0}\right)$ and $y_{k}=\tau^{k}\left(y_{0}\right)$. Then $\left(x_{k}, y_{k}\right) \in \widetilde{\mathcal{R}}$ for $0 \leq k<L$. Define $d^{\tau}$ on $\widetilde{\mathcal{R}}^{\tau}$ by

$$
d^{\tau}(x, y)=\sum_{k=0}^{L-1} d\left(x_{k}, y_{k}\right) .
$$

Since $\left(x_{k}, y_{k}\right)$ depends continuously on $(x, y), d^{\tau}$ is continuous. It is clear that $d^{\tau}$ satisfies the cocycle identity $d^{\tau}(x, y)+d^{\tau}(y, z)=d^{\tau}(x, z)$, for $(x, y),(y, z) \in \widetilde{\mathcal{R}}^{\tau}$. Since $(x, y) \in \mathcal{R}^{\tau}$ if and only if $\left(x_{k}, y_{k}\right) \in \mathcal{R}$ for all $0 \leq k<L$, we have $d^{\tau}(x, y) \geq 0$ if and only if $(x, y) \in \mathcal{R}^{\tau}$.

Conversely, suppose that $\mathcal{T}^{\tau}$ is analytic with cocycle $d^{\tau}$. If $(x, y) \in \widetilde{\mathcal{R}}$ then $(\pi(x), \pi(y)) \in \widetilde{\mathcal{R}}^{\tau}$, and if $(x, y) \in \mathcal{R}$ then $(\pi(x), \pi(y)) \in \mathcal{R}^{\tau}$. Thus a cocycle $d$ for $\mathcal{T}$ can be defined by $d(x, y)=d^{\tau}(\pi(x), \pi(y))$. That $d$ satisfies the cocycle condition is clear. Also, by Remark 3.1.2, $d^{-1}(0)=\{(x, x): x \in X\}$. The continuity of $d$ follows from that of $\pi$ and $d^{\tau}$.

5.2 Lemma. Let $(\Omega, \Phi)$ be an inductive system, and let $\Omega^{\prime} \subset \Omega$ be of infinite cardinality. If $\mathcal{T}(\Omega, \Phi)$ is analytic, then $\mathcal{T}\left(\Omega^{\prime}, \Phi\right)$ is analytic.

Proof. By Proposition 3.4.3, the semigroupoid $\mathcal{R}^{\tau}\left(\Omega^{\prime}, \Phi\right)$ of the $\tau$-invariant subalgebra $\mathcal{T}^{\tau}\left(\Omega^{\prime}, \Phi\right)$ can be realized as a subset of $\mathcal{R}^{\tau}(\Omega, \Phi)$. Thus, the restriction to $\mathcal{R}^{\tau}\left(\Omega^{\prime}, \Phi\right)$ of a cocycle for $\mathcal{T}^{\tau}(\Omega, \Phi)$ is a cocycle for $\mathcal{T}^{\tau}\left(\Omega^{\prime}, \Phi\right)$. The conclusion now follows from the previous lemma.

5.3 Theorem. Let $(\Omega, \Phi)$ be an inductive system. Then $\mathcal{T}(\Omega, \Phi)$ is analytic if and only if $\Omega$ is order-isomorphic to the integers, the positive integers, or the negative integers.

Proof. If $\Omega=\mathbb{Z}, \mathcal{T}^{\tau}(\Omega, \Phi)$ is the alternation algebra, if $\Omega=\mathbb{Z}^{+}, \mathcal{T}^{\tau}(\Omega, \Phi)$ is the refinement algebra, and if $\Omega=\mathbb{Z}^{-}, \mathcal{T}^{\tau}(\Omega, \Phi)$ is the standard algebra. These are analytic. Now apply Lemma 5.1 to conclude $\mathcal{T}(\Omega, \Phi)$ is analytic.

Conversely, if $\Omega$ is not one of these three, $\Omega$ contains a subset $\Omega^{\prime}$ which is orderisomorphic with either $\left\{\omega_{0}\right\}+\mathbb{Z}^{-}$or $\mathbb{Z}^{+}+\left\{\omega_{0}\right\}$. The following lemmas show that in either case, $\mathcal{T}\left(\Omega^{\prime}, \Phi\right)$ is not analytic. The conclusion derives from Lemma 5.2.

5.4 Lemma. If $\Omega$ is order-isomorphic with $\left\{\omega_{0}\right\}+\mathbb{Z}^{-}$and $L=1, \mathcal{T}(\Omega, \Phi)$ is not analytic.

Proof. Let $\eta$ be the dimension function of the system $(\Omega, \Phi)$. As in Section 3 , the maximal ideal space $X$ of the diagonal is a product space $\prod_{\omega \in \Omega}[\eta(\omega)]$, and an ordered 
pair $(x, y) \in X \times X$ belongs to the groupoid $\widetilde{\mathcal{R}}$ iff $x_{\omega}=y_{\omega}$ for all but finitely many $\omega \in \Omega$. Furthermore, $(x, y) \in \widetilde{\mathcal{R}}$ belongs to the semigroupoid $\mathcal{R}$ if $x$ precedes $y$ in the lexicographic order. In this case we write $x \prec y$.

Let $v \in \mathcal{T}(\Omega, \Phi)$ be the matrix unit whose graph is $\widehat{v}=\left\{(x, y): x_{-1}=0, y_{-1}=\right.$ $1, x_{\omega_{0}}=y_{\omega_{0}}=0$, and $x_{n}=y_{n}$ for $\left.n \in \mathbb{Z}^{-},|n|>1\right\}$. Let $\underline{0} \in X$ be the element $\underline{0}_{\omega}=0, \omega \in \Omega$, and define sequences $x^{(n)}, y^{(n)} \in X$ by

$$
x_{\omega}^{(n)}= \begin{cases}0, & \omega \neq n, \\ 1, & \omega=n,\end{cases}
$$

and

$$
y_{\omega}^{(n)}= \begin{cases}0, & \omega \neq n \text { or }-1 \\ 1, & \omega=n \text { or }-1\end{cases}
$$

for $n=-2,-3, \ldots$. Then we have

$$
\begin{aligned}
\underline{0} \prec x^{(-2)} & \prec y^{(-2)} \prec x^{(-3)} \prec y^{(-3)} \prec \ldots \\
& \prec x^{(n)} \prec y^{(n)} \prec x^{(n-1)} \prec \ldots .
\end{aligned}
$$

Now $\left(x^{(n)}, y^{(n)}\right) \in \widehat{v} \subset \mathcal{R}$, and $\left(y^{(n)}, x^{(n-1)}\right) \in \mathcal{R}$. $\widehat{v}$ is a compact subset of $\mathcal{R}$ disjoint from the diagonal. If $\mathcal{T}$ were analytic with cocycle $d$, then $d$ would have a positive minimum value, say $\varepsilon$, on $\widehat{v}$. Furthermore, as $d$ is nonnegative on $\mathcal{R}$,

$$
\begin{aligned}
d\left(\underline{0}, x^{(n)}\right) & =d\left(\underline{0}, x^{(-2)}\right)+d\left(x^{(-2)}, y^{(-2)}\right) \\
& +d\left(y^{(-2)}, x^{(-3)}\right)+d\left(x^{(-3)}, y^{(-3)}\right) \\
& +\cdots+d\left(x^{(n+1)}, y^{(n+1)}\right)+d\left(y^{(n+1)}, x^{(n)}\right) \\
& \geq(|n|-2) \varepsilon .
\end{aligned}
$$

Let $x^{(0)} \in X$ be the element

$$
x_{\omega}^{(0)}= \begin{cases}0, & \omega \in \mathbb{Z}^{-}, \\ 1, & \omega=\omega_{0} .\end{cases}
$$

Then $x^{(n)} \prec x^{(0)}$ for all $n \in \mathbb{Z}^{-}$, and we have

$$
\begin{aligned}
d\left(\underline{0}, x^{(0)}\right) & =d\left(\underline{0}, x^{(n)}\right)+d\left(x^{(n)}, x^{(0)}\right) \\
& \geq(|n|-2) \varepsilon .
\end{aligned}
$$

As $|n|$ could be arbitrarily large, $d\left(\underline{0}, x^{(0)}\right)$ is not a real number. Thus, no such cocycle $d$ can exist.

5.5 Lemma. If $\Omega$ is order-isomorphic with $\mathbb{Z}^{+}+\left\{\omega_{0}\right\}$ and $L=1$, then $\mathcal{T}(\Omega, \Phi)$ is not analytic.

Proof. Suppose $\mathcal{T}(\Omega, \Phi)$ is analytic with cocycle $d$. Let $v$ be the matrix unit whose graph is $\widehat{v}=\left\{(x, y) \in \mathcal{R}: x_{n}=y_{n}, n \in \mathbb{Z}^{+}, x_{\omega_{0}}=0, y_{\omega_{0}}=1\right\}$. This is a compact subset of $\mathcal{R}$, disjoint from the diagonal, so that $d$ has a strictly positive minimum 
value, say $\varepsilon$, on $\widehat{v}$. Let $\underline{0} \in X$ be the element $\underline{0}_{\omega}=0, \omega \in \Omega$, and define sequences $x^{(n)}, y^{(n)}, n \geq 1$, in $X$ by

$$
\begin{gathered}
x_{\omega}^{(n)}= \begin{cases}0, & \omega \neq n, \\
1, & \omega=n,\end{cases} \\
y_{\omega}^{(n)}= \begin{cases}1, & \omega=n \text { or } \omega_{0}, \\
0, & \text { otherwise }\end{cases}
\end{gathered}
$$

Then $\left(x^{(n)}, y^{(n)}\right) \in \widehat{v} \subset \mathcal{R}$, and $\left(y^{(n+1)}, x^{(n)}\right) \in \mathcal{R}$. Thus

$$
\begin{aligned}
d\left(\underline{0}, x^{(1)}\right) & =d\left(\underline{0}, x^{(n)}\right)+d\left(x^{(n)}, y^{(n)}\right) \\
& +d\left(y^{(n)}, x^{(n-1)}\right)+d\left(x^{(n-1)}, y^{(n-1)}\right) \\
& +\cdots+d\left(y^{(2)}, x^{(1)}\right) \geq(n-1) \varepsilon
\end{aligned}
$$

This shows that no such real-valued cocycle $d$ exists.

\section{The Jacobson RADical}

Given an inductive system $(\Omega, \Phi)$, let $\mathcal{J}=\mathcal{J}(\Omega, \Phi)$ denote the Jacobson radical of the TAF algebra $\mathcal{T}(\Omega, \Phi)$, and $\mathcal{J}^{\tau}$ the Jacobson radical of the $\tau$-fixed subalgebra $\mathcal{T}^{\tau}(\Omega, \Phi)$. Power has provided a description of the Jacobson radical in the case $L=1$ [Pr 2], which in turn is based on Donsig's characterization of the radical [D]. We will utilize Power's result below.

6.1 Lemma. $E^{\tau}(\mathcal{J}) \subset \mathcal{J}^{\tau}$.

Proof. Recall that the Jacobson radical is a characteristic ideal; that is, it is invariant under any automorphism of $\mathcal{T}$. Thus if $x \in \mathcal{J}, \tau(x), \ldots, \tau^{L-1}(x)$ are in $\mathcal{J}$ as well, and so is $E^{\tau}(x)$. Thus, for any $y \in \mathcal{T}, y E^{\tau}(x)$ is quasinilpotent, and in particular this holds for any $y \in \mathcal{T}^{\tau}$. But then $E^{\tau}(x) \in \mathcal{J}^{\tau}$.

6.2 Proposition. For any matrix unit $v \in \mathcal{T}, v \in \mathcal{J}$ if and only if $E^{\tau}(v) \in \mathcal{J}^{\tau}$.

Proof. One direction has just been done. Suppose now that $v$ is a matrix unit of $\mathcal{T}$ and that $E^{\tau}(v)$ belongs to $\mathcal{J}^{\tau}$. Say $v=e_{\underline{i}, \underline{j}}^{(F, k)}$ for some finite set $F \subset \Omega, k \in[L]$, and $\underline{i}, \underline{j} \in I_{F}$. Replacing $v$ by $\tau^{-k}(v)$, we may suppose that $k=0$. This will not change $E^{\tau}(v)$, and $v \in \mathcal{J}$ iff $\tau^{-k}(v) \in \mathcal{J}$.

Let $p_{0}$ be the central projection onto $\mathfrak{A}_{F, 0}$, and $p_{k}=\tau^{k}\left(p_{0}\right)$ the central projection onto $\mathfrak{A}_{F, k}, k \in[L]$. Let $w=L \cdot E^{\tau}(v)$. We claim that for any $z \in \mathcal{T}, p_{0} z p_{0} w$ is quasinilpotent. Now $E^{\tau}\left(p_{0} z p_{0}\right) w$ is quasinilpotent, as $w \in \mathcal{J}^{\tau}$. Writing $z_{k}=\tau^{k}(z)$, we have

$$
\begin{aligned}
E^{\tau}\left(p_{0} z p_{0}\right) w & =\frac{1}{L}\left(\sum_{k \in[L]} p_{k} z_{k} p_{k}\right)\left(\sum_{l \in[L]} e_{\underline{i}, \underline{j}}^{(F, l)}\right) \\
& =\frac{1}{L} \sum_{k \in[L]} p_{k} z_{k} p_{k} e_{\underline{i}, \underline{j}}^{(F, k)}
\end{aligned}
$$

So,

$$
L^{m}\left\|\left[E^{\tau}\left(p_{0} z p_{0}\right) w\right]^{m}\right\|=\max _{0 \leq k<L}\left\|\left[p_{k} z_{k} p_{k} e_{\underline{i}, \underline{j}}^{(F, k)}\right]^{m}\right\| .
$$


It follows that, for $z \in \mathcal{T}, p_{0} z v=p_{0} z e_{\underline{i}, \underline{j}}^{(F, 0)}=p_{0} z_{0} p_{0} e_{\underline{i}, \underline{j}}^{(F, 0)}$ is quasinilpotent.

Now write $z v=\sum_{k \in[L]} p_{k} z e_{\underline{i}, \underline{j}}^{(F, 0)}$. Then $(z v)^{m}=\sum_{k \in[L]} p_{k} z e_{\underline{i}, \underline{j}}^{(F, 0)}\left(p_{0} z e_{\underline{i}, \underline{j}}^{(F, 0)}\right)^{m-1}$. Thus $\left\|(z v)^{m}\right\| \leq L\|z\|\left\|\left(p_{0} z e_{\underline{i}, \underline{j}}^{(F, 0)}\right)^{m-1}\right\|$. It follows from the above paragraph that $z v$ is quasinilpotent, and hence $v \in \mathcal{J}$.

From the proposition, every matrix unit in $\mathcal{J}^{\tau}$ also belongs to $\mathcal{J}$. As $\mathcal{J}^{\tau}$ is a norm-closed ideal in $\mathcal{T}^{\tau}$, and in particular a norm-closed $\mathcal{D}^{\tau}$-bimodule, $\mathcal{J}^{\tau}$ is the closed span of the matrix units it contains. We obtain

6.3 Corollary. $E^{\tau}(\mathcal{J})=\mathcal{J}^{\tau}$.

6.4 Remark. It is not true that $\left(E^{\tau}\right)^{-1}\left(\mathcal{J}^{\tau}\right)$ equals $\mathcal{J}$. For example, if $L=2$, the diagonal element $d=e_{\underline{i}}^{(F, 0)}-e_{\underline{i}}^{(F, 1)}\left(F \subset \Omega, \underline{i} \in I_{F}\right)$ satisfies $E^{\tau}(d)=0 \in \mathcal{J}^{\tau}$, but $d \notin \mathcal{J}$.

Let $(\Omega, \Phi)$ be an inductive system. If $\Omega^{\prime}$ is an (infinite) subset of $\Omega$ we can (and will) identify the algebras $\mathcal{D}\left(\Omega^{\prime}, \Phi\right), \mathcal{T}\left(\Omega^{\prime}, \Phi\right)$ and $\mathfrak{A}\left(\Omega^{\prime}, \Phi\right)$ canonically with subalgebras of $\mathcal{D}(\Omega, \Phi), \mathcal{T}(\Omega, \Phi)$, and $\mathfrak{A}(\Omega, \Phi)$ respectively. We define a conditional expectation $E_{\Omega^{\prime}}: \mathfrak{A}(\Omega, \Phi) \rightarrow \mathcal{D}\left(\Omega^{\prime}, \Phi\right)^{c}$, the commutant of $\mathcal{D}\left(\Omega^{\prime}, \Phi\right)$ in $\mathfrak{A}(\Omega, \Phi)$, as follows: Let $\left\{F_{n}\right\}_{n=1}^{\infty}$ be a sequence of finite subsets of $\Omega^{\prime}, F_{1} \subset F_{2} \subset \ldots$, with $\bigcup_{n=1}^{\infty} F_{n}=\Omega^{\prime}$. Define $E_{n}: \mathfrak{A} \rightarrow \mathcal{D}_{F_{n}}^{c}$ by

$$
E_{n}(a)=\sum_{k \in[L] \underline{i} \in I_{n}} e_{\underline{i}}^{(n, k)} a e_{\underline{i}}^{(n, k)}
$$

where $\left\{e_{\underline{i}}^{(n, k)}: \underline{i} \in I_{n}\right\}$ are diagonal matrix units for $\mathfrak{A}_{F_{n}, k}$. The $\left\{E_{n}\right\}_{n=1}^{\infty}$ converge in the pointwise-norm topology, and the limit is denoted by $E_{\Omega^{\prime}}(a)$. The proofs of these statements are similar to the proof of existence of the Stratila-Voiculescu conditional expectation $E=E_{\Omega}$. (See [PPW1, Lemma 2.1].)

6.5 Lemma. $E_{\Omega^{\prime}} E^{\tau}=E^{\tau} E_{\Omega^{\prime}}$.

Proof. We have

$$
\begin{aligned}
E_{n} E^{\tau}(a) & =\frac{1}{L} \sum_{k \in[L]} E_{\Omega^{\prime}}\left(\tau^{k}(a)\right)=\frac{1}{L} \sum_{k \in[L]} \sum_{l \in[L]} \sum_{\underline{i} \in I_{n}} e_{\underline{i}}^{(n, l)} \tau^{k}(a) e_{\underline{i}}^{(n, l)} \\
& =\frac{1}{L} \sum_{k \in[L]} \sum_{l \in[L] \underline{i} \in I_{n}} \sum_{\underline{i}}^{(n, l+k)} \tau^{k}(a) e_{\underline{i}}^{(n, l+k)} \text { as } l+k \text { is } \bmod L \\
& =\frac{1}{L} \sum_{k \in[L]} \tau^{k}\left(\sum_{l \in[L]} \sum_{\hat{i} \in I_{n}} e_{\underline{i}}^{(n, l)} a e_{\underline{i}}^{(n, l)}\right)=\frac{1}{L} \sum_{k \in[L]} \tau^{k} E_{n}(a) \\
& =E^{\tau} E_{n}(a)
\end{aligned}
$$

Thus, $E_{\Omega^{\prime}} E^{\tau}(a)=E^{\tau} E_{\Omega^{\prime}}(a)$.

6.6 Theorem. Let $(\Omega, \Phi)$ be an inductive system. Decompose $\Omega$ into an order sum $\Omega=\Omega_{0}+\Omega_{1}$, where $\Omega_{0}$ is the maximal well-ordered initial segment. Then the Jacobson radical

$$
\mathcal{J}=\left\{a \in \mathcal{T}: E_{\Omega_{0}}(a)=0\right\}
$$


Proof. As $\mathcal{J}$ is a norm-closed $\mathcal{D}$-bimodule, it is the closed span of the matrix units it contains ([PPW1, Theorem 2.2].) As $E_{\Omega_{0}}$ is continuous, it is enough to show that a matrix unit $v \in \mathcal{T}$ belongs to $\mathcal{J}$ iff $E_{\Omega_{0}}(v)=0$. We can apply Power's result [Pr 2, Theorem 3] to the $\tau$-fixed subalgebra $\mathcal{T}^{\tau}$ to express $\mathcal{J}^{\tau}=\mathcal{T}^{\tau} \cap \operatorname{ker} E_{\Omega_{0}}$. By Proposition 6.2, a matrix unit $v \in \mathcal{T}$ belongs to $\mathcal{J}$ iff $E^{\tau}(v) \in \mathcal{J}^{\tau}$. Thus, $v \in \mathcal{J}$ iff $E_{\Omega_{0}}\left(E^{\tau}(v)\right)=0$.

Set $\mathcal{I}=\left\{a \in \mathcal{T}: E_{\Omega_{0}}(a)=0\right\}$. As $d_{1} E_{\Omega_{0}}(a) d_{2}=E_{\Omega_{0}}\left(d_{1} a d_{2}\right), a \in \mathfrak{A}, d_{1}, d_{2} \in \mathcal{D}$, $\mathcal{I}$ is a norm-closed $\mathcal{D}$-bimodule, and so it is the norm-closed span of the matrix units it contains. Thus, to show $\mathcal{I}=\mathcal{J}$, it suffices to show they contain the same set of matrix units of $\mathcal{T}$.

If $v$ is a matrix unit in $\mathcal{I}$, then by Lemma $6.5, E_{\Omega_{0}} E^{\tau}(v)=E^{\tau} E_{\Omega_{0}}(v)=0$, so $v \in \mathcal{J}$. On the other hand, if $v \in \mathcal{J}$, say $v=e_{\underline{i}, \underline{j}}^{(F, k)}$ for some finite $F \subset \Omega, k \in$ $[L], \underline{i}, \underline{j} \in I_{F}$, then $E^{\tau}(v)=\sum_{l \in[L]} e_{\underline{i}, \underline{j}}^{(F, l)}$. If $p_{k}$ is the central projection onto the factor $\mathfrak{A}_{F, k}$ in $\mathfrak{A}_{F}$, then

$$
\begin{aligned}
0 & =E_{\Omega_{0}} E^{\tau}(v) \\
\Rightarrow 0 & =E_{\Omega_{0}}\left(\sum_{l \in[L]} e_{\underline{i}, \underline{j}}^{(F, l)}\right) \\
\Rightarrow 0 & =p_{k} E_{\Omega_{0}}\left(\sum_{l \in[L]} e_{\underline{i}, \underline{j}}^{(F, l)}\right)=E_{\Omega_{0}}\left(p_{k} \sum_{l \in[L]} e_{\underline{i}, \underline{j}}^{(F, l)}\right) \\
\Rightarrow 0 & =E_{\Omega_{0}}\left(e_{\underline{i}, \underline{j}}^{(F, k)}\right)=E_{\Omega_{0}}(v) .
\end{aligned}
$$

Hence $v \in \mathcal{J}$.

6.7 Corollary. $\mathcal{T}(\Omega, \Phi)$ is semisimple if and only if $\Omega$ has no first element.

\section{Outer Automorphisms}

We begin by establishing the connection between the outer automorphism group of a TAF algebra, and the automorphism group of the associated semigroupoid. All automorphisms studied in this and the next section are assumed to be isometric.

Let $\mathcal{T}$ be TAF in an AF algebra $\mathfrak{A}$, and suppose $\left\{\mathfrak{A}_{n}\right\}_{n=1}^{\infty}$ is an increasing chain of finite dimensional $C^{*}$-algebras with $\mathfrak{A}=\overline{\cup \mathfrak{A}_{n}}$ such that $\mathcal{T}=\overline{\bigcup\left(\mathcal{T} \cap \mathfrak{A}_{n}\right)}$, and $\mathcal{T} \cap \mathfrak{A}_{n}$ is maximal triangular in $\mathfrak{A}_{n}$. Let a system of matrix units for $\mathfrak{A}_{n}$ be given, $n \geq 1$, such that each matrix unit in $\mathfrak{A}_{n}$ is a sum of matrix units in $\mathfrak{A}_{n+1}$. Let $\alpha$ be an automorphism of $\mathcal{T}$. As is known, $\alpha$ extends to an automorphism of the AF algebra $\mathfrak{A}$, preserving the diagonal $\mathcal{D}=\mathcal{T} \cap \mathcal{T}^{*}$. Now $\alpha$ induces a map $\widehat{\alpha}$ on the groupoid $\widetilde{\mathcal{R}}$, mapping the support of a matrix unit $v$ (which is a compact open $G$-set) to a compact open $G$-set, which is expressible as a (finite) disjoint union of support sets of matrix units, say $\widehat{\alpha}: \widehat{v} \rightarrow \bigcup \widehat{v}_{i}$. If $\alpha_{0}$ is defined by $\alpha_{0}(v)=\sum v_{i}$, then $\alpha_{0}$ is seen to be an automorphism of $\mathcal{T}$, and $\alpha(v) \alpha_{0}(v) * \in \mathcal{D}$, for any matrix unit $v$ [PPW1]. Since $\alpha_{0}$ maps matrix units to sums of matrix units, we can replace the sequence $\left\{\mathfrak{A}_{n}\right\}_{i=1}^{\infty}$ by a subsequence so that $\alpha_{0}\left(\mathfrak{A}_{n}\right) \subset \mathfrak{A}_{n+1}, n=1,2, \ldots$. 
7.1 Lemma. With notation as above, there is a sequence $\left\{u^{(n)}\right\}_{n=1}^{\infty}$ of unitaries in $\mathcal{D}$ such that

$$
\alpha(a)=\lim _{n} A d\left(u^{(n)}\right) \alpha_{0}(a)
$$

for any $a \in \mathfrak{A}$.

Proof. We note that for $d \in \mathcal{D}, \alpha(d)=\alpha_{0}(d)$. For each $n$, let $\left\{e_{i j}^{(n, k)}: 1 \leq\right.$ $k \leq N(n), 1 \leq i, j \leq M(n, k)\}$ be a system of matrix units of $\mathfrak{A}_{n}$ such that $e_{i i}^{(n, k)} \in \mathcal{D}$ for all $i$ and $k$. Let $u_{i j}^{(n, k)}=\alpha\left(e_{i j}^{(n, k)}\right) \alpha_{0}\left(e_{j i}\right)$. Then $u_{i j}^{(n, k)} \in \mathcal{D}$ and $u^{(n)}=\sum_{k=1}^{N(n)} \sum_{i=1}^{M(n, k)} u_{i 1}^{(n, k)}$ is a unitary in $\mathcal{D}$, and we have

$$
\begin{aligned}
A d\left(u^{(n)}\right) \alpha_{0}\left(e_{i^{\prime} j^{\prime}}^{\left(n, k^{\prime}\right)}\right) & =\left(\sum_{k=1}^{N(n)} \sum_{i=1}^{M(n, k)} u_{i 1}^{(n, k)}\right) \alpha_{0}\left(e_{i^{\prime} j^{\prime}}^{\left(n, k^{\prime}\right)}\right)\left(\sum_{k=1}^{N(n)} \sum_{i=1}^{M(n, k)} u_{i 1}^{(n, k)}\right)^{*} \\
& =\alpha\left(e_{i^{\prime} 1}^{\left(n, k^{\prime}\right)}\right) \alpha_{0}\left(e_{1 i^{\prime}}^{\left(n, k^{\prime}\right)}\right) \alpha_{0}\left(e_{i^{\prime} j^{\prime}}^{\left(n, k^{\prime}\right)}\right) \alpha_{0}\left(e_{j^{\prime} 1}^{\left(n, k^{\prime}\right)}\right) \alpha\left(e_{1 j^{\prime}}^{\left(n, k^{\prime}\right)}\right) \\
& =\alpha\left(e_{i^{\prime} 1}^{\left(n, k^{\prime}\right)}\right) \alpha_{0}\left(e_{11}^{\left(n, k^{\prime}\right)}\right) \alpha\left(e_{1 j^{\prime}}^{\left(n, k^{\prime}\right)}\right) \\
& =\alpha\left(e_{\left.i^{\prime} k^{\prime}\right)}^{\left(n, k^{\prime}\right)} \alpha\left(e_{11}^{\left(n, k^{\prime}\right)}\right) \alpha\left(e_{1 j^{\prime}}^{\left(n, k^{\prime}\right)}\right)\right. \\
& =\alpha\left(e_{i^{\prime} j^{\prime}}^{\left(n, k^{\prime}\right)}\right)
\end{aligned}
$$

Thus, for every $a \in \bigcup \mathfrak{A}_{n}, \alpha(v)=\lim _{n} \operatorname{Ad}\left(u^{(n)}\right) \alpha_{0}(a)$, as the sequence is eventually constant. As $\mathfrak{A}=\overline{\cup \mathfrak{A}_{n}}$, the conclusion follows.

Two automorphisms $\alpha, \beta$ of $\mathfrak{A}$ are said to be inner equivalent if there is a sequence $\left\{u^{(n)}\right\}_{n=1}^{\infty}$ of unitaries in $\mathcal{D}$ such that

$$
\alpha(a)=\lim _{n} A d\left(u^{(n)}\right) \beta(a),
$$

for any $a \in \mathfrak{A}$. Suppose for each $n$, a system of matrix units is chosen for $\mathfrak{A}_{n}$. The above lemma shows that every automorphism $\alpha$ is inner equivalent to an $\alpha_{0}$ which maps matrix units in $\mathfrak{A}_{n}$ to sums of matrix units in $\mathfrak{A}_{n+1}$.

Let $\mathcal{R}$ be the semigroupoid of the TAF algebra $\mathcal{T}$. An automorphism $\widehat{\alpha}$ of $\mathcal{R}$ is a homeomorphism of $\mathcal{R}$ which preserves composition:

$$
\widehat{\alpha}(x, z)=\widehat{\alpha}(x, y) \widehat{\alpha}(y, z)
$$

for all pairs $(x, y),(y, z) \in \mathcal{R} \subset X \times X$.

7.2 Proposition. i) $A$ map $\widehat{\alpha}$ is an automorphism of $\mathcal{R}$ if and only if $\widehat{\alpha}(x, y)=$ $(h(x), h(y))$ for some homeomorphism $h$ of $X$ such that $(h(x), h(y)) \in \mathcal{R}$ for all $(x, y) \in \mathcal{R}$.

ii) Every semigroupoid automorphism $\widehat{\alpha}$ induces an automorphism $\alpha_{0}$ of $\mathcal{T} . \alpha_{0}$ depends on the choice of the system of matrix units.

iii) Every automorphism $\alpha$ of $\mathcal{T}$ induces an automorphism $\widehat{\alpha}$ of the semigroupoid $\mathcal{R}$. The map $\alpha \mapsto \widehat{\alpha}, \operatorname{Aut}(\mathcal{T}) \rightarrow \operatorname{Aut}(\mathcal{R})$, is onto.

iv) Let $\alpha, \beta \in \operatorname{Aut}(\mathcal{T})$; then $\widehat{\alpha}=\widehat{\beta}$ iff $\alpha, \beta$ are approximately inner equivalent.

Proof. i) Let $\widehat{\alpha}$ be an automorphism of $\mathcal{R}$. 7.1 implies that $\widehat{\alpha}$ maps the diagonal $\{(x, x): x \in X\}$ to itself. Thus, there exists a homeomorphism $h$ of $X$ such that 
$\widehat{\alpha}(x, x)=(h(x), h(x))$ for all $x \in X$. For every $(x, y) \in \mathcal{R}$, we have

$$
\begin{aligned}
\widehat{\alpha}(x, y) & =\widehat{\alpha}(x, x) \widehat{\alpha}(x, y)=(h(x), h(x)) \widehat{\alpha}(x, y) \\
& =\widehat{\alpha}(x, y) \widehat{\alpha}(y, y)=\widehat{\alpha}(x, y)(h(y), h(y)) .
\end{aligned}
$$

Therefore, $\widehat{\alpha}(x, y)=(h(x), h(y))$.

Conversely, if $h$ is a homeomorphism of $X$ satisfying $(h(x), h(y)) \in \mathcal{R}$ if and only if $(x, y) \in \mathcal{R}$, then $\widehat{\alpha}(x, y)=(h(x), h(y))$ defines an automorphism $\widehat{\alpha}$ of $\mathcal{R}$.

ii) Let $\widehat{\alpha}$ be an automorphism of $\mathcal{R}$. An automorphism $\alpha_{0}$ of $\mathcal{R}$ can be defined as follows: if $v$ is a matrix unit in $\bigcup \mathcal{T} \cap \mathfrak{A}_{n}$, its support $\widehat{v}$ is a compact-open $G$ set; hence so is $\widehat{\alpha}(\widehat{v})$. Thus $\widehat{\alpha}(\widehat{v})=\bigcup_{i} \widehat{v}_{i}$ for matrix units $\left\{v_{i}\right\}$ in $\bigcup \mathcal{T} \cap \mathfrak{A}_{n}$. Set $\alpha_{0}(v)=\sum v_{i}$.

iii) Let $\alpha$ be an automorphism of $\mathcal{T}$; then $\alpha$ restricts to an automorphism of the diagonal $\mathcal{D}$, hence induces a homeomorphism $h$ on $X$. Let $\widehat{\alpha}(x, y)=(h(x), h(y))$. Then $\widehat{\alpha}$ is an automorphism of $\mathcal{R}$. The second part follows from ii) and the fact that $\widehat{\alpha_{0}}=\widehat{\alpha}$.

iv) $\widehat{\alpha}=\widehat{\beta}$ iff $\alpha_{0}=\beta_{0}$, where $\alpha_{0}, \beta_{0}$ are as in ii). But by Lemma 7.1 , this holds iff $\alpha, \beta$ are approximately inner equivalent.

\subsection{Corollary. $\operatorname{Out}(\mathcal{T}) \cong \operatorname{Aut}(R(\mathcal{T}))$}

Returning to the framework of lexicographic inductive systems, suppose $(\Omega, \Phi)$ is such a system where $\Omega$ has order type $\mathbb{Z}^{+}$and the AF algebra $\mathfrak{A}(\Omega, \Phi)$ is simple. In that case $\mathcal{T}(\Omega, \Phi)$ is a nest algebra (Cor. 4.6). Let $\alpha \in A u t(\mathcal{T})$. Since Lat $\mathcal{T}$ is a maximal nest $(4.5$, Remark 3$), \alpha($ Lat $\mathcal{T})=$ Lat $\mathcal{T}$. Now $\left.\alpha\right|_{\mathfrak{A} \tau}$ maps the UHF $C^{*}$ algebra $\mathfrak{A}^{\tau}$ onto the UHF algebra $\alpha\left(\mathfrak{A}^{\tau}\right)$. Thus $\alpha: A l g \mathcal{L} \cap \mathfrak{A}^{\tau} \rightarrow \operatorname{Alg} \mathcal{L} \cap \alpha\left(\mathfrak{A}^{\tau}\right)(\mathcal{L}=$ Lat $\mathcal{T}=$ Lat $\mathcal{T}^{\tau}$ ) is trace-preserving by [Pe Wa, Prop. 2.16]. Since $p, q \in \mathcal{L}, p \leq q$ implies $\alpha(p) \leq \alpha(q)$; trace-preserving means $\alpha(p)=p, p \in \mathcal{L}$. As $\mathcal{D}^{\tau}=C^{*}(\mathcal{L}), \widehat{\alpha}$ induces the identity on $X^{\tau}=\widehat{\mathcal{D}^{\tau}}$, and so $\widehat{\alpha}=i d$ on $\widetilde{\mathcal{R}}^{\tau}$. Now let $x^{(0)} \in X$ be a minimal point. Then $\widehat{\alpha}\left(x^{(0)}\right)$ is also a minimal point of $X$, of which there are $L \geq 1$. (By abuse of notation, we write $\widehat{\alpha}$ to denote the homeomorphism on $X$, as well as the automorphism of $\mathcal{R}$.) Thus, $\alpha\left(x^{(0)}\right)=\tau^{k}\left(x^{(0)}\right)$ for some integer $k \in[L]$. Let $\beta=\tau^{-k} \alpha$. Then $\beta \in \operatorname{Aut}(\mathcal{T})$. Since $\widehat{\beta}$ maps orbits in $\widetilde{\mathcal{R}}$ to orbits, $\widehat{\beta}$ maps the orbit of $x^{(0)}$ to itself. Since $\widehat{\alpha}$ induces the identity on $\mathcal{R}^{\tau}$, so does $\widehat{\beta}$. Thus if $x=(k, \underline{i}) \in$ $\operatorname{orbit}\left(x^{(0)}\right)$, then $\widehat{\beta}(x)=y=\left(k^{\prime}, \underline{i}\right)$ for some $k^{\prime} \in[L]$. But since $y \in \operatorname{orbit}\left(x^{(0)}\right)$, $k^{\prime}=k$, so $\widehat{\beta}=i d$ on $\operatorname{orbit}\left(x^{(0)}\right)$. Thus, $\widehat{\alpha}=\widehat{\tau}^{k}$. We summarize this result as:

7.4 Example. If $(\Omega, \Phi)$ is a lexicographic inductive system with $\mathfrak{A}(\Omega, \Phi)$ a simple $C^{*}$-algebra, and if $\Omega$ has order type $\mathbb{Z}^{+}$, then

$$
\operatorname{Out}(\mathcal{T}(\Omega, \Phi)) \cong \mathbb{Z} / L \mathbb{Z} \text {. }
$$

Next, consider systems $(\Omega, \Phi)$ where $\Omega$ has order type $\mathbb{Z}^{-}$. In this case the embeddings $\Phi_{F, G}$ are of standard type [Po Wa], and so the TAF algebra $\mathcal{T}(\Omega, \Phi)$ is $\mathbb{Z}$-analytic (cf. Theorem 5.3). The orbit of any minimal point in $X=\widehat{\mathcal{D}}$ is ordered as $\mathbb{Z}^{+}$. Fix a minimal point $x^{(0)} \in X$. If $\alpha \in \operatorname{Aut}(\mathcal{T})$, then $\widehat{\alpha}\left(x^{(0)}\right)$ is another minimal point, so $\widehat{\alpha}\left(x^{(0)}\right)=\widehat{\tau}^{k}\left(\left(x^{(0)}\right)\right.$. Let $\beta=\tau^{-k} \alpha$. Then not only does $\beta$ map $x^{(0)}$ to itself, but it maps the orbit of $x^{(0)}$ to itself, preserving the ordering. Thus, the $k^{t h}$ successor of $x^{(0)}$ in $X$ is mapped to itself. If the orbit of $x^{(0)}$ is dense in $X$, which will be the case iff $\mathfrak{A}(\Omega, \Phi)$ is simple, then $\widehat{\beta}=i d$. 
7.5 Example. If $(\Omega, \Phi)$ is a lexicographic inductive system with $\mathfrak{A}(\Omega, \Phi)$ a simple $C^{*}$-algebra, and if $\Omega$ has order type $\mathbb{Z}^{-}$, then

$$
\text { Out }(\mathcal{T}(\Omega, \Phi)) \cong \mathbb{Z} / L \mathbb{Z}
$$

\section{Automorphisms of SEmigroupoids $\mathcal{R}(\Omega, \Phi), \Omega$ A DENSE ORDERING}

Let $\Omega$ be a dense linear ordering with dimension function $\eta$, and $X=\prod_{\omega \in \Omega}[\eta(\omega)]$. Let $\mathcal{R} \subset X \times X$ be the lexicographic semigroupoid as defined in 1.1. If $\Omega^{\prime}$ is another dense ordering with dimension function $\eta^{\prime}$ and $X^{\prime}, \mathcal{R}^{\prime}$ are analogous, Power [Pr 3] showed that $\mathcal{R}, \mathcal{R}^{\prime}$ are isomorphic as semigroupoids if and only if there is an order isomorphism $\varphi: \Omega \rightarrow \Omega^{\prime}$ such that $\eta^{\prime}(\varphi(\omega))=\eta(\omega), \omega \in \Omega$. In that case, if $\alpha_{\varphi}: X \rightarrow X^{\prime}$ is the homeomorphism defined by $\alpha_{\varphi}(x)=y$, where $y_{\varphi(\omega)}=x_{\omega}$, then $\alpha_{\varphi} \times \alpha_{\varphi}: \mathcal{R} \rightarrow \mathcal{R}^{\prime}$ is a semigroupoid isomorphism. One can ask if every isomorphism $\alpha: \mathcal{R} \rightarrow \mathcal{R}^{\prime}$ is of the form $\alpha_{\varphi}$. Equivalently, is every automorphism of $\mathcal{R}$ of the form $\alpha_{\varphi}$, where $\varphi$ is on order-preserving, dimension-preserving bijection of $\Omega$ ? The answer to this question (which is not addressed in [Pr 3]) is affirmative.

Of course $\mathcal{R}$ is the semigroupoid of a lexicographic TAF (actually TUHF) algebra $\mathcal{T}(\Omega, \Phi)$ with $L(\Phi)=1$. However, $\mathcal{T}$ will play no role in the discussion. We break the convention of the previous section and let $\alpha$ (rather than $\widehat{\alpha}$ ) denote a homeomorphism of $X$ such that $\alpha \times \alpha$ is an automorphism of $\mathcal{R}$. For $v \in \Omega$, set $x^{(v)}$ the element of $X$ with

$$
x_{\omega}^{(v)}= \begin{cases}0, & \omega \leq v, \\ \eta(\omega)-1, & \omega>v,\end{cases}
$$

and $\mathcal{X}=\left\{x^{(v)}: v \in \Omega\right\}$. $\mathcal{X}$ can be characterized in order-topological terms, from which it follows that $\alpha(\mathcal{X})=\mathcal{X}$. This is the first step in the proof of the main result.

In keeping with the notation of previous sections, write $y \prec x$ (or, $x \succ y$ ) if $(y, x) \in \mathcal{R} . x \sim y$ if $(y, x) \in \widetilde{\mathcal{R}}$; that is, if either $(y, x) \in \mathcal{R}$ or $(x, y) \in \mathcal{R}$. A set $Y \subset X$ is decreasing if $x \in Y$ and $y \prec x$ imply $y \in Y$. If $Y$ is decreasing, then so is the (topological) closure of $Y, \operatorname{cl}(Y)$ ([PPW2, Proposition 3.3]). $\mathcal{O}^{-}(x)=\{y \in$ $X: y \prec x\}$ denotes the one-sided orbit, and its closure, $\operatorname{cl}\left(\mathcal{O}^{-}(x)\right)$, is decreasing.

8.1 Lemma. Let $x \in X$. Then $x \in \mathcal{X}$ if and only if

i) for any $y \prec x, \operatorname{cl}\left(\mathcal{O}^{-}(y)\right)=\operatorname{cl}\left(\mathcal{O}^{-}(x)\right)$;

ii) for any $y \succ x, y \neq x, \operatorname{cl}\left(\mathcal{O}^{-}(y)\right)$ strictly contains $\operatorname{cl}\left(\mathcal{O}^{-}(x)\right)$;

iii) there is a unique $y \in X, y \notin \operatorname{cl}\left(\mathcal{O}^{-}(x)\right)$, such that $\operatorname{cl}\left(\mathcal{O}^{-}(y)\right)=\{y\} \cup$ $\operatorname{cl}\left(\mathcal{O}^{-}(x)\right)$.

Proof. First we show that any $x \in \mathcal{X}$ satisfies these conditions. Observe that $\operatorname{cl}\left(\mathcal{O}^{-}\left(x^{(v)}\right)=\left\{x \in X: x_{\omega}=0, \omega \leq v\right\}\right.$.

For i), let $y \prec x^{(v)}$. It is enough to show $x^{(v)} \in \operatorname{cl}\left(\mathcal{O}^{-}(y)\right)$. Let $F \subset \Omega$ be a finite subset. We will find $z \in \mathcal{O}^{-}(y)$ such that $z_{\omega}=x_{\omega}^{(v)}, \omega \in F$. Of course, if $\omega \leq v$ for all $\omega \in F$, then $y_{\omega}=0=x_{\omega}^{(v)}$, for all $\omega \in F$, so we can take $z=y$. Otherwise, let $\omega_{0}=\min \{\omega \in F: \omega>v\}$. Set $G=\left\{\omega: y_{\omega} \neq x_{\omega}^{(v)}\right\}$; note that if $\omega \in G, \omega>v$. If $G=\emptyset$, then $y=x^{(v)}$, and there is nothing to prove. Let $\omega_{1}=\min \{\omega: \omega \in G\}$ and 
$\omega_{2}=\min \left\{\omega_{0}, \omega_{1}\right\}$, and $\omega_{3}$ any point of $\Omega$ in the interval $v<\omega<\omega_{2}$. Define $z$ by

$$
z_{\omega}= \begin{cases}\eta(\omega)-1, & v<\omega, \omega \neq \omega_{3}, \\ 0, & \omega=\omega_{3}, \\ 0, & \omega \geq v .\end{cases}
$$

Then $z$ has the required properties.

For ii), let $y \succ x^{(v)}$ and $y \neq x^{(v)}$; then there is some $\omega \leq v$ with $y_{\omega}>0$. Thus, $y \notin \operatorname{cl}\left(\mathcal{O}^{-}\left(x^{(v)}\right)\right)$, and hence $\operatorname{cl}\left(\mathcal{O}^{-}(y)\right)$ strictly contains $\operatorname{cl}\left(\mathcal{O}^{-}(x)\right)$.

For iii) define $y \in X$ as follows:

$$
y_{\omega}= \begin{cases}1, & \omega=v \\ 0, & \omega \neq v .\end{cases}
$$

Then $y \notin \operatorname{cl}\left(\mathcal{O}^{-}\left(x^{(v)}\right)\right)$, and $\operatorname{cl}\left(\mathcal{O}^{-}(y)\right)=\{y\} \cup \operatorname{cl}\left(\mathcal{O}^{-}\left(x^{(v)}\right)\right)$. It is also evident there is no other element with this property.

Conversely, suppose $x \in X$ satisfies i), ii) and iii). Let $\Omega_{0}=\left\{\omega: x_{\omega^{\prime}}=0\right.$ for all $\left.\omega^{\prime} \leq \omega\right\}, \Omega_{1}=\Omega \backslash \Omega_{0}$. Then $\Omega=\Omega_{0}+\Omega_{1}$, order sum.

If $\Omega_{1}$ has a smallest element, say $v$, then let $y=\left(y_{\omega}\right)$ be given by

$$
y_{\omega}= \begin{cases}x_{\omega}, & \omega \neq v \\ x_{\omega}-1, & \omega=v\end{cases}
$$

then $y \prec x$ and $\operatorname{cl}\left(\mathcal{O}^{-}(y)\right)$ does not contain $x$. Thus, $\Omega_{1}$ has no smallest element.

Clearly, $\operatorname{cl}\left(\mathcal{O}^{-}(x)\right) \subseteq\left\{z \in X: z_{\omega}=0\right.$, for all $\left.\omega \in \Omega_{0}\right\}$. On the other hand, suppose $z \in X$ and $z_{\omega}=0$ for all $\omega \in \Omega_{0}$. Let $F \subset \Omega$ be finite. If $F \cap \Omega_{1}=\emptyset$, then we have $x_{\omega}=z_{\omega}$ for all $\omega \in F$. Suppose $F \cap \Omega_{1} \neq \emptyset$. We can choose $\omega_{0} \in \Omega_{1}$ such that $\omega_{0}<\omega$ for all $\omega \in F \cap \Omega_{1}$ and $x_{\omega_{0}}>0$. Define

$$
y_{\omega}= \begin{cases}x_{\omega_{0}}-1, & \omega=\omega_{0}, \\ z_{\omega}, & \omega \in F, \\ x_{\omega}, & \omega \notin F \cup\left\{\omega_{0}\right\} .\end{cases}
$$

Then $y=\left(y_{\omega}\right) \prec x$ and $y_{\omega}=z_{\omega}$, for all $\omega \in F$. Hence, $\operatorname{cl}\left(\mathcal{O}^{-}(x)\right)=\left\{z \in X: z_{\omega}=\right.$ 0 , for all $\left.\omega \in \Omega_{0}\right\}$.

Next, we will show that $x_{\omega}=\eta(\omega)-1$, for all $\omega \in \Omega_{1}$. Suppose for some $\omega_{1} \in \Omega_{1}, x_{\omega_{1}}<\eta\left(\omega_{1}\right)-1$. Define $y$ by

$$
y_{\omega}= \begin{cases}x_{\omega}, & \omega \neq \omega_{1}, \\ \eta\left(\omega_{1}\right)-1, & \omega=\omega_{1} .\end{cases}
$$

Then $y \succ x, y \neq x$. The argument in the previous paragraph shows that $\operatorname{cl}\left(\mathcal{O}^{-}(y)\right)$ $=\left\{z \in X: z_{\omega}=0\right.$, for all $\left.\omega \in \Omega_{0}\right\}=\operatorname{cl}\left(\mathcal{O}^{-}(x)\right)$, a contradiction to ii).

We have that $x_{\omega}=0, \omega \in \Omega_{0}, x_{\omega}=\eta(\omega)-1, \omega \in \Omega_{1}$, and $\Omega_{1}$ has no least element. Finally, we show $\Omega_{0}$ has a largest element. Let $y \notin \operatorname{cl}\left(\mathcal{O}^{-}(x)\right)$. Then there is some $\omega_{0} \in \Omega_{0}, y_{\omega_{0}}>0$. If $\Omega_{0}$ has no largest element, there is $\omega^{\prime} \in \Omega_{0}, \omega_{0}<\omega^{\prime}$. Define $z$ by

$$
z_{\omega}= \begin{cases}0, & \omega=\omega_{0}, \\ 1, & \omega=\omega^{\prime}, \\ y_{\omega}, & \omega \neq \omega_{0}, \omega^{\prime} .\end{cases}
$$


Then $y \neq z, z \in \operatorname{cl}\left(\mathcal{O}^{-}(y)\right)$. But $z \notin \operatorname{cl}\left(\mathcal{O}^{-}(x)\right)$. Thus, iii) is violated. Therefore, $\Omega_{0}$ has a largest element, say $v$, and $x=x^{(v)}$.

8.2 Lemma. The collection of sets of the form $\operatorname{cl}\left(\mathcal{O}^{-}(x)\right)(x \in X)$ is linearly ordered by inclusion.

Proof. For $x \in X$, set $\Omega_{0}(x)=\left\{\omega \in \Omega: x_{\omega^{\prime}}=0\right.$, for all $\left.\omega^{\prime} \leq \omega\right\}$. For $x \neq y \in X$, if $\Omega_{0}(x)$ is properly contained in $\Omega_{0}(y)$, then $\operatorname{cl}\left(\mathcal{O}^{-}(x)\right)$ properly contains $c l\left(\mathcal{O}^{-}(y)\right)$. So we may assume $\Omega_{0}(x)=\Omega_{0}(y)$. Let $\Omega_{1}=\left\{\omega \in \Omega: x_{\omega^{\prime}}=y_{\omega^{\prime}}\right.$, for all $\left.\omega^{\prime} \leq \omega\right\}$ and $\Omega_{2}=\Omega \backslash \Omega_{1} \neq \emptyset$. If $\Omega_{2}$ has a least element, say $\omega_{1}$, then $\operatorname{cl}\left(\mathcal{O}^{-}(x)\right) \subset \operatorname{cl}\left(\mathcal{O}^{-}(y)\right)$ (resp., $\left.\operatorname{cl}\left(\mathcal{O}^{-}(y)\right) \subset \operatorname{cl}\left(\mathcal{O}^{-}(x)\right)\right)$ if $x_{\omega_{1}}<y_{\omega_{1}}$ (resp., $\left.y_{\omega_{1}}>x_{\omega_{1}}\right)$. If $\Omega_{2}$ has no least element, then either

i) for every $\omega \in \Omega_{2}$ there exist an infinite number of $\omega^{\prime}$ in $\Omega_{2}$ such that $\omega^{\prime}<\omega$ and $x_{\omega^{\prime}}>0$, or

ii) for every $\omega \in \Omega_{2}$ there exist an infinite number of $\omega^{\prime}$ in $\Omega_{2}$ such that $\omega^{\prime}<\omega$ and $y_{\omega^{\prime}}>0$.

If i) holds, then $\operatorname{cl}\left(\mathcal{O}^{-}(y)\right) \subseteq \operatorname{cl}\left(\mathcal{O}^{-}(x)\right)$. Otherwise, $\operatorname{cl}\left(\mathcal{O}^{-}(x)\right) \subseteq \operatorname{cl}\left(\mathcal{O}^{-}(y)\right)$.

Set $x^{(v, i)} \in X$ to be the element

$$
x_{\omega}^{(v, i)}=\left\{\begin{array}{ll}
0, & \omega<v \\
i, & \omega=v, \\
\eta(\omega)-1, & \omega>v,
\end{array} \quad(i \in[\eta(v)]),\right.
$$

and $X_{(v, i)}=\operatorname{cl}\left(\mathcal{O}^{-}\left(x^{(v, i)}\right)=\left\{x \in X: x_{v} \leq i, x_{\omega}=0, \omega<v\right\}\right.$.

8.3 Lemma. Let $v \in \Omega$. In the collection of closed orbits $\operatorname{cl}\left(\mathcal{O}^{-}(x)\right), x \succ x^{(v)}$, linearly ordered by inclusion, the orbit $\operatorname{cl}\left(\mathcal{O}^{-}\left(x^{(v, i)}\right)\right)$ is the immediate successor of $\operatorname{cl}\left(\mathcal{O}^{-}\left(x^{(v, i-1)}\right)\right), 0<i<\eta(v) . \quad \operatorname{cl}\left(\mathcal{O}^{-}\left(x^{(v, i)}\right)\right)$ has no immediate successor if $i=\eta(v)-1$.

Proof. Note the assertion does not imply that $x^{(v, i)}$ is the immediate successor of $x^{(v, i-1)}$ in the orbit of $x^{(v)}$. Clearly $X_{v, i} \supsetneqq X_{v, i-1}$. Suppose $x \in X, x^{(v, i-1)} \prec x \prec$ $x^{(v, i)}$. If $x \neq x^{(v, i-1)}$, then $x_{v}=i$. But then $\operatorname{cl}\left(\mathcal{O}^{-}(x)\right)=X_{v, i}$. This proves the first statement. Now suppose $x \succ x^{(v, i)}$, where $i=\eta(v)-1, x \neq x^{(v, i)}$. Then there is some $w<v$ with $x_{w}>0$. Let $\omega_{0}$ satisfy $w<\omega_{0}<v$ and $x_{\omega_{0}}=0$. Such $\omega_{0}$ exists since the set $\{\omega: w<\omega<v\}$ is infinite. If $y \in X$ is given by

$$
y_{\omega}= \begin{cases}0, & \omega=w \\ 1, & \omega=\omega_{0} \\ x_{w}, & \text { otherwise }\end{cases}
$$

then $\operatorname{cl}\left(\mathcal{O}^{-}\left(x^{(v, i)}\right)\right)=X_{v, i} \varsubsetneqq \operatorname{cl}\left(\mathcal{O}^{-}(y)\right) \varsubsetneqq \operatorname{cl}\left(\mathcal{O}^{-}(x)\right)(i=\eta(v)-1)$. Thus the set of closed orbits $c l\left(\mathcal{O}^{-}(x)\right), x \succ x^{(v, i)}, x \neq x^{(v, i)}(i=\eta(v)-1)$, has no least element.

8.4 Corollary. Let $\alpha$ be a homeomorphism of $X$ such that $\alpha \times \alpha$ is an automorphism of $\mathcal{R}$. Then there is an order-isomorphism $\varphi: \Omega \rightarrow \Omega$ such that $\alpha\left(x^{(v)}\right)=x^{\varphi(v)}$, and $\eta(\varphi(v))=\eta(v), v \in \Omega$.

Proof. If $x=x^{(v)}, v \in \Omega$, so $x$ satisfies i), ii), iii) of Lemma 1, then necessarily $\alpha(x)$ satisfies these conditions. Thus, there is a bijection $\varphi: \Omega \rightarrow \Omega$ such that $\alpha\left(x^{(v)}\right)=x^{\varphi(v)}$. As $x \prec y$ implies $\alpha(x) \prec \alpha(y), \varphi$ is an order-isomorphism. Now the chain $X_{(v, 0)} \subset X_{(v, 1)} \subset \cdots \subset X_{(v, \eta(v)-1)}$ is the maximal chain which contains 
$X_{(v, 0)}=\operatorname{cl}\left(\mathcal{O}^{-}\left(x^{(v)}\right)\right)$ such that each member of the chain has either an immediate successor or immediate predecessor in the collection $\left\{\operatorname{cl}\left(\mathcal{O}^{-}(x)\right): x \succ x^{(v)}\right\}$. Thus, the cardinality of the chain is invariant under $\alpha$; i.e., $\eta(\varphi(v))=\eta(v), v \in \Omega$.

8.5 Corollary. Set $X^{(v, i)}=\left\{x \in X_{(v, i)}: x_{v}=i\right\}$. Then $\alpha\left(X^{(v, i)}\right)=X^{(\varphi(v), i)}$ for $v \in \Omega$ and $i \in[\eta(v)])$.

Proof. If $i=0, X^{(v, 0)}=X_{(v, 0)}=\operatorname{cl}\left(\mathcal{O}^{-}\left(x^{(v)}\right)\right)$, and by the previous corollary this is mapped by $\alpha$ onto $\operatorname{cl}\left(\mathcal{O}^{-}\left(x^{(\varphi(v))}\right)=X_{\varphi(v), 0}\right.$. Likewise $\alpha\left(X_{v, i}\right)=X_{(\varphi(v), i)}$. But if $i>0$, then $X^{(v, i)}=X_{(v, i)} \backslash X_{(v, i-1)}$, and so

$$
\begin{aligned}
\alpha\left(X^{(v, i)}\right) & =\alpha\left(X_{(v, i)}\right) \backslash \alpha\left(X_{(v, i-1)}\right) \\
& =X_{(\varphi(v), i)} \backslash X_{(\varphi(v), i-1)} \\
& =X^{(\varphi(v), i)} .
\end{aligned}
$$

Let $\alpha_{\varphi}$ denote the homeomorphism $\alpha_{\varphi}(x)=y$, where $y_{\varphi(\omega)}=x_{\omega}, \omega \in \Omega$. Then $\alpha_{\varphi} \times \alpha_{\varphi}$ is an automorphism of $\mathcal{R}$. The remainder of this section is devoted to showing that $\alpha_{\varphi}$ and $\alpha$ agree on a certain dense subset of $X$, and hence by continuity must agree everywhere. To this end, given $\omega_{1}<\cdots<\omega_{n}$ in $\Omega$ and integers $i_{t} \in \eta\left(\omega_{t}\right), 1 \leq t \leq n$, set

$$
X^{\left(\omega_{1}, i_{1}\right), \ldots\left(\omega_{n}, i_{n}\right)}=\left\{x \in X: x_{\omega}=\left\{\begin{array}{ll}
i_{t}, & \omega=\omega_{t}, 1 \leq t \leq n, \\
0, & \omega<\omega_{n}, \omega \neq \omega_{t}, 1 \leq t \leq n
\end{array}\right\} .\right.
$$

\subsection{Lemma.}

$$
\alpha\left(X^{\left(\omega_{1}, i_{1}\right), \ldots,\left(\omega_{n}, i_{n}\right)}\right)=X^{\left(\varphi\left(\omega_{1}\right), i_{1}\right), \ldots,\left(\varphi\left(\omega_{n}\right), i_{n}\right)} .
$$

Proof. The proof will be by induction on $n$. For $n=1$, this is Corollary 8.5. Since the statement holds if $\alpha=\alpha_{\varphi}$, replace $\alpha$ by $\beta=\alpha_{\varphi}^{-1} \alpha$. We need to show $\beta$ leaves $X^{\left(\omega_{1}, i_{1}\right), \ldots,\left(\omega_{n}, i_{n}\right)}$ invariant. Fix a positive integer $N$, and assume the result holds for $n \leq N$. That is, assume that for any $n$ points $\omega_{1}<\cdots<\omega_{n}(n \leq N)$ in $\Omega$ and integers $i_{t} \in\left[\eta\left(\omega_{t}\right)\right], 1 \leq t \leq n, \beta$ leaves $X^{\left(\omega_{1}, i_{1}\right), \ldots,\left(\omega_{n}, i_{n}\right)}$ invariant. Suppose $N+1$ points $\omega_{1}<\cdots<\omega_{n}$ in $\Omega$ and $i_{t} \in\left[\eta\left(\omega_{t}\right)\right], 1 \leq t \leq n=N+1$, are given. Set $Y=X^{\left(\omega_{1}, i_{1}\right), \ldots,\left(\omega_{n-1}, i_{n-1}\right)}$. By the induction hypothesis, $\beta(Y)=Y$. Let $\Omega^{\prime}=\left\{\omega>\omega_{n-1}\right\}$ and $X^{\prime}=\prod_{\omega \in \Omega^{\prime}}[\eta(\omega)]$. The projection $\zeta: X \rightarrow X^{\prime}$ restricts to $\left.\zeta\right|_{Y}: Y \rightarrow X$, and $\left.\zeta\right|_{Y}$ is one-to-one. Thus, $\beta^{\prime}: X^{\prime} \rightarrow X^{\prime}, \beta^{\prime}(\zeta(y))=\zeta(\beta(y)), y \in Y$, defines a homeomorphism of $X^{\prime}$, which gives an automorphism for the semigroupoid $\mathcal{R}^{\prime}$ associated with $X^{\prime}$. Thus, the analysis of 8.1-8.5 can be applied to $\left(X^{\prime}, \beta^{\prime}\right)$. In particular, there is an order isomorphism $\psi$ of $\Omega^{\prime}$, preserving dimensions, such that $\beta^{\prime}\left(X_{(v, i)}^{\prime}\right)=X_{(\psi(v), i)}^{\prime}, v \in \Omega^{\prime}, i \in[\eta(v)]$. Equivalently, $\beta\left(Y_{(v, i)}\right)=Y_{(\psi(v), i)}, v>$ $\omega_{n-1}, i \in[\eta(v)]$. Let $u^{(v, i)}$ be the unique maximal element of $Y_{(v, i)}$ : so

$$
u_{\omega}^{(v, i)}= \begin{cases}i_{t}, & \omega=\omega_{t}, 1 \leq t<n, \\ i, & \omega=v, \\ 0, & \omega<v, \omega \neq \omega_{t}, 1 \leq t<n, \\ \eta(\omega)-1, & \omega>v\end{cases}
$$


Then $\beta$ maps $u^{(v, i)}$ to the maximal element of $Y_{(\psi(v), i)}, u^{(\psi(v), i)}$. Define $z \in X$,

$$
z_{\omega}= \begin{cases}u_{\omega}^{(v, i)}, & \omega \neq \omega_{1}, \\ 0, & \omega=\omega_{1} .\end{cases}
$$

Then $z \in X^{\left(\omega_{2}, i_{2}\right), \ldots,\left(\omega_{n-1}, i_{n-1}\right),(v, i)}$. By the induction hypothesis, this set is mapped to itself by $\beta$. Furthermore, $z$ is the maximal element of $X^{\left(\omega_{2}, i_{2}\right), \ldots,\left(\omega_{n-1}, i_{n-1}\right),(v, i)}$, so $\beta(z)=z$. Now $z, u^{(v, i)}$ are equivalent, since they differ in only one coordinate. Thus, $\beta\left(u^{(v, i)}\right)$ is equivalent to $u^{(v, i)}$. However, if either $\psi(v)>v$ or $\psi(v)<v$, there will be infinitely many coordinates on which $u^{(v, i)}, u^{(\psi(v), i)}$ differ. Thus $\psi=i d$. Taking $(v, i)=\left(\omega_{n}, i_{n}\right)$ completes the induction.

Let $x\left(\left(\omega_{1}, i_{1}\right), \ldots\left(\omega_{n}, i_{n}\right)\right)$ denote the element of $X$ whose $\omega_{t}^{\text {th }}$ coordinate is $i_{t}, 1 \leq t \leq n$, and whose other coordinates are all zero. Note that the element $x\left(\left(\omega_{1}, i_{1}\right), \ldots\left(\omega_{n}, i_{n}\right)\right)$ is the unique minimal element of $X^{\left(\omega_{1}, i_{1}\right), \ldots\left(\omega_{n}, i_{n}\right)}$. Thus if $\beta$ is an automorphism of $\mathcal{R}$ leaving $X^{\left(\omega_{1}, i_{1}\right), \ldots,\left(\omega_{n}, i_{n}\right)}$ invariant, $\beta$ must fix the point $x\left(\left(\omega_{1}, i_{1}\right), \ldots\left(\omega_{n}, i_{n}\right)\right)$. But the set of points with finitely many nonzero coordinates is dense in $X$. Thus $\beta=i d$. This proves

\subsection{Theorem. $\alpha=\alpha_{\varphi}$.}

8.8 Remark. For $L>1$, the structure of $A u t(\mathcal{R})$ is more complicated. Let $\operatorname{Hom}(\Omega)$ be the set of all order preserving bijections $\phi$ of $\Omega$ such that $\eta(\omega)=\eta(\phi(\omega))$ and $\nu(\omega, i)=\nu(\phi(\omega), i)$ for all $\omega \in \Omega$ and $i \in[\eta(\omega)]$. Then we can define $\alpha_{\phi} \in A u t(\mathcal{R})$ by $\alpha_{\phi}((k, x))=(k, y)$, where $y_{\omega}=x_{\phi(\omega)}$. Also, $\tau \in \operatorname{Aut}(\mathcal{R})$. A natural question to ask is: Is $\operatorname{Aut}(\mathcal{R})$ generated by $\tau$ and $\left\{\alpha_{\phi}, \phi \in \operatorname{Hom}(\Omega)\right\}$ ? The answer is no.

For example, let $\Omega$ be the set of rational numbers and $L=2$. Let $a, b \in \Omega$ and $a<b$. Define $\eta(\omega)=2$ for all $\omega \in \Omega$, and, for $i \in[\eta(\omega)], \nu(\omega, i)=0$ if $a \leq \omega \leq b$ and $\nu(\omega, i)=i$ if $\omega<a$ or $\omega>b$. Define $\phi_{0}, \phi_{1}: \Omega \rightarrow \Omega$ by $\phi_{0}(\omega)=\omega$ for all $\omega \in \Omega$, and

$$
\phi_{1}(\omega)= \begin{cases}\omega & \text { if } \omega<a \text { or } \omega>b, \\ (\omega+a) / 2 & \text { if } a \leq \omega \leq(a+2 b) / 3 \\ 2 \omega-b & \text { if }(a+2 b) / 3 \leq \omega \leq b .\end{cases}
$$

Then $\alpha\left(\left(k, x_{\omega}\right)\right)=\left(k, y_{\omega}\right)$, where $y_{\omega}=x_{\phi_{k}(\omega)}$ defines an automorphism of $\mathcal{R}$ which is not generated by $\tau$ and $\left\{\alpha_{\phi}: \phi \in \operatorname{Hom}(\Omega)\right\}$.

Note that in this example, $\mathcal{A}(\Omega, \Phi)$ is simple.

\section{REFERENCES}

[D] A-P. Donsig, Semisimple triangular AF algebras, J. Functional Analysis 111 (1993), 323-349. MR 94b:46084

[DH] A-P. Donsig and A. Hopenwasser, Order preservation in limit algebras, J. Functional Analysis 133 (1995), 342-394. MR 96k:46099

[HPS] R. Herman, I. Putnam and C. Skau, Ordered Bratteli diagrams, dimension groups and topological dynamics, Internat. J. Math. 3 (1992), 827-864. MR 94f:46096

[Hu] S. Hu, Homotopy Theory, Academic Press (1959). MR 21:5186

[MS] P.S. Muhly and B. Solel, Subalgebras of groupoid $C^{*}$-algebras, J. für die reine und angew. Math. 402 (1989), 41-75. MR 90m:46098

[Pe Wa] J.R. Peters and B.H. Wagner, Triangular AF algebras and nest subalgebras of UHF algebras, J. Operator Theory 25 (1991), 79-123. MR 94c:46116

[PPW1] J.R. Peters, Y.T. Poon and B.H. Wagner, Triangular AF algebras, J. Operator Theory 23 (1990), 81-114. MR 91h:46102 
[PPW2] J.R. Peters, Y.T. Poon and B.H. Wagner, Analytic TAF algebras, Can. J. Math., Vol. 45 (5), (1993), 1009-1031. (Correction: Vol. 46 (2) (1994), 395-6.). MR 94m:46113a,b

[Po Wa] Y.T. Poon and B.H. Wagner, $\mathbb{Z}$-analytic TAF algebras and dynamical systems, Houston J. Math. 19 (1993), 181-199. MR 95f:46113

[Pr 1] S.C. Power, On the outer automorphism groups of triangular alternation limit algebras, J. Functional Analysis 113 (1993), 462-471. MR 95g:47065

[Pr 2] Infinite lexicographic products of triangular algebras, Bull. London Math. Soc. 27 (1995), 273-277. MR 96e:47047

[Pr 3] Lexicographic semigroupoids, Ergodic Theory Dynamical Systems 16 (1996), 365-377. MR 97d:47050

[R] J.G. Rosenstein, Linear Orderings, Academic Press, 1982. MR 84m:06001

[Wa] B. Wagner, Triangular AF algebras induced by lexicographic orders, preliminary report.

Department of Mathematics, Iowa State University, Ames, Iowa 50011-2064

E-mail address: peters@iastate.edu

E-mail address: ytpoon@iastate.edu 